\title{
Multi-soft theorems in gauge theory from MHV diagrams
}

\section{George Georgiou}

Institute of Nuclear and Particle Physics, National Center for Scientific Research Demokritos, Aghia Paraskevi 15310, Athens, Greece

E-mail: georgiou@inp.demokritos.gr

ABSTRACT: In this work we employ the MHV technique to show that scattering amplitudes with any number of consecutive soft particles behave universally in the multi-soft limit in which all particles go soft simultaneously. After identifying the diagrams which give the leading contribution we give the general rules for writing down compact expressions for the multi-soft factor of $m$ gluons, $k$ of which have negative helicity. We explicitly consider the cases where $k=1$ and 2 . In $\mathcal{N}=4 \mathrm{SYM}$, the multi-soft factors of 2 scalars or 2 fermions forming a singlet of SU(4) $R$-symmetry, and $m-2$ positive helicity gluons are derived. The special case of the double-soft limit gives an amplitude whose leading divergence is $1 / \delta^{2}$ and not $1 / \delta$ as in the case of 2 scalars or 2 fermions that do not form a singlet under $\mathrm{SU}(4)$. The construction based on the analytic supervertices allows us to obtain simple expressions for the triple-soft limit of 1 scalar and 2 positive helicity fermions, as well as for the quadrapole-soft limit of 4 positive helicity fermions, in a singlet configuration.

KEYwords: Scattering Amplitudes, Supersymmetric gauge theory, Extended Supersymmetry

ARXiv EPrint: 1505.08130 


\section{Contents}

1 Introduction 1

2 Gluonic multi-soft limits and the CSW method 3

2.1 Gluonic multi-soft factor with one negative helicity gluon 3

$\begin{array}{lll}2.2 & \text { Rules for generic gluonic multi-soft factor } & 7\end{array}$

2.3 Gluonic multi-soft factor with two negative helicity gluons 8

3 Multi-soft limits in $N=4$ SYM from the analytic supervertex $\quad 12$

$\begin{array}{ll}3.1 & \text { Iterating the analytic supervertex } \\ 3.2\end{array}$

3.2 Multi-soft limit of 2 scalars or 2 fermions forming a singlet and $m-2$ positive $\begin{array}{ll}\text { helicity gluons } & 14\end{array}$

$\begin{array}{lll}3.3 & \text { Double-soft limit of } 2 \text { scalars forming a singlet } & 16\end{array}$

$\begin{array}{ll}3.4 & \text { Double-soft limit of } 2 \text { fermions forming a singlet } \\ \end{array}$

3.5 Multi-soft limit of ( 2 fermions +1 scalar) and ( 4 fermions) forming a singlet 17

4 Conclusions

\section{Introduction}

On-shell scattering amplitudes is one of the most important observables calculated in quantum field theory. ${ }^{1}$ The complete knowledge of the scattering matrix specifies the theory, at least at the perturbative level. Moreover, scattering amplitudes of gauge field theories frequently exhibit structures and symmetries which are not at all apparent from the conventional Lagrangian formulation of the theory. ${ }^{2}$

In particular, the soft behaviour of the $\mathrm{S}$ matrix of a theory generically reflects symmetries of this theory. One of the most important examples of this fact is Weinberg's soft theorem $[1,2]$ which constrains the universal behaviour of the leading divergence of the S-matrix in the soft limit by use of Ward identities. The universal behaviour of the subleading terms in the momentum of the soft particle for gluons and gravitons have been also established in [3, 4]. Another example, is that of the soft behaviour of Goldstone bosons in the case of a spontaneous broken symmetry. Taking the momentum of the Goldstone boson to approach zero leads to vanishing amplitude, a fact known as Adler's zero [5]. If one takes the double-soft limit of 2 scalars the resulting amplitude is non vanishing and can be related to some underlying symmetry of the theory. For example, in the case of $\mathcal{N}=8$ supergravity the double-soft limit reveals the $E_{7(7)}$ symmetry algebra of the scalar

\footnotetext{
${ }^{1}$ Another important class of observables is that of correlation functions of gauge invariant operators. For some recent progress on this front see [15-20] and references therein.

${ }^{2}$ For detailed reviews on this and other related subjects see [21-23].
} 
moduli space [6]. Similar double-soft theorems for spin 1/2 particles and the restrictions that these may impose on supergravity counter terms have been also discussed in [26]. Furthermore, recently the subleading soft graviton theorems $[3,4]$ was argued to be related to large gauge transformations (diffeomorphisms in the case of gravity) [7-9]. The relevant asymptotic symmetry group should be the $B M S_{4}$ algebra $[10,11]$ or some extension of it in other than 4 space-time dimensions [7-9, 12-14, 32-35]. The effect of loop corrections to the soft theorems was also considered in [27-31]. More recently, the double-soft theorems for scalars and fermions have been extended to include the double-soft limits of gluons and gravitons $[24,25]$. Further studies on the soft limit of the S matrix in gauge theories, gravity and string theory can be found in [36-46].

In almost all the studies mentioned above the soft theorems were established through the BCFW recursion relations [47, 48] in 4 dimensions or through the CHY formula in the case of arbitrary dimensions [49]. In this work we will follow a slightly different route and employ the CSW (or scalar graph) method [50]. This method was originally proposed as an alternative to Feynman diagrammatics and was inspired by the weak-to-weak duality between $\mathcal{N}=4$ SYM theory and the topological B model with target space the Calabi-Yau supermanifold $C P^{3 \mid 4}[51]$. This method was firstly proven to hold for tree-level gluonic amplitudes. Subsequently, it was proven to hold for all tree-level amplitudes in gauge theories with any amount of supersymmetry and for any number of colours $[52,53]$. The validity of the method at the loop level was also shown for the maximally supersymmetric theory, as well as in theories with less supersymmetry $[54-58,63] .{ }^{3}$ The advantage of this approach is that it will allow us to easily prove multi-soft theorems involving an arbitrary number of soft particles. Furthermore, it will also allow us to obtain compact expressions for the leading singularity of amplitudes involving any number of soft gluons, fermions and/or scalars.

The plan of the paper is the following. In section 2 we discuss how the CSW method can be applied to the simultaneous multi-soft limit of an arbitrary number of gluons. To be more precise the multi-soft limit that we will be considering is the following:

$$
p_{i}=\delta q_{i}, \quad \lambda_{p_{i}}^{\alpha}=\sqrt{\delta} \lambda_{q_{i}}^{\alpha} \equiv \sqrt{\delta} \lambda_{i}^{\alpha}, \quad \tilde{\lambda}_{p_{i}}^{\dot{\alpha}}=\sqrt{\delta} \tilde{\lambda}_{q_{i}}^{\dot{\alpha}} \equiv \sqrt{\delta} \tilde{\lambda}_{i}^{\dot{\alpha}}, \quad \delta \rightarrow 0, \quad i=1, \ldots, m
$$

That is we take the momenta of $\mathrm{m}$ consecutive gluons to scale to zero simultaneously. In particular, in section 2.1 we prove the multi-soft theorem and derive the multi-soft factor of $\mathrm{m}$ soft gluons one of which is of negative helicity. In section 2.2 we give the general rules from which one can obtain the multi-soft factor of $m$ soft gluons $k$ of which have negative helicity. As an example, in section 2.3 we apply these rules to write down the expression for the case of 2 negative and $m-2$ positive helicity gluons. In all cases the result takes the form of the multi-soft limit of $\mathrm{m}$ positive helicity gluons times a factor depending on the negative helicity ones. In section 3 we consider the multi-soft limit in $\mathcal{N}=4 \mathrm{SYM}$ theory. After reviewing the scalar graph approach based on the analytic supervertices of the theory, we proceed to consider the multi-soft limit of 2 scalars or 2 fermions forming a singlet under the SU(4) R-symmetry group and $m-2$ positive helicity gluons. In sections

\footnotetext{
${ }^{3}$ The equivalence of the MHV amplitudes with the expectation value of polygonal Wilson loops at both weak and strong coupling was also the subject of intense investigations [59-62].
} 
3.3 and 3.4 we focus on the double soft limit of 2 scalars and 2 fermions respectively. We obtain the soft factor whose leading divergence is $1 / \delta^{2}$ and not $1 / \delta$ as in the case of 2 scalars or 2 fermions that do not form a singlet under SU(4). In section 3.5, we derive the expressions for the multi-soft function of ( 2 fermions +1 scalar) and ( 4 fermions) forming a singlet. In all cases the amplitude has a leading divergence of $1 / \delta^{m}$ in the soft limit (2.1). Finally, in section 4 we conclude.

Before closing this section a couple of comments are in order. The objects that we will be dealing with will be the kinematic parts of the colour-stripped scattering amplitudes. Furthermore, we will be suppressing the overall momentum conservation factor of the amplitudes. Finally, the spinor conventions the we will adopt are those of [52].

\section{Gluonic multi-soft limits and the CSW method}

One way to obtain compact expressions for purely gluonic amplitudes with any number of particles at tree level is by employing the CSW formalism [50]. In this section we show how one can use this technique to get compact expressions for the multi-soft functions multiplying the "hard" amplitude when any number of adjacent gluons are taken soft simultaneously, namely

$$
p_{i}=\delta q_{i}, \quad \lambda_{p_{i}}^{\alpha}=\sqrt{\delta} \lambda_{q_{i}}^{\alpha} \equiv \sqrt{\delta} \lambda_{i}^{\alpha}, \quad \tilde{\lambda}_{p_{i}}^{\dot{\alpha}}=\sqrt{\delta} \tilde{\lambda}_{q_{i}}^{\dot{\alpha}} \equiv \sqrt{\delta} \tilde{\lambda}_{i}^{\dot{\alpha}}, \quad \delta \rightarrow 0, \quad i=1, \ldots, m .
$$

When all these gluons are of the same helicity one can show that the multi-soft factor is the product of $\mathrm{m}$ single soft factors as if the particles were taken soft one by one. This is no longer true when the multi-soft limit involves gluons of different helicities [24, 25]. In this note we will restrict ourselves to the leading term in the $1 / \delta$ expansion of the universal multi-soft factor $S_{m}$. The first step consists in identifying the set of MHV diagrams which contribute in the soft limit. As we will see only a limited number of MHV diagrams contribute in the soft limit (2.1).

\subsection{Gluonic multi-soft factor with one negative helicity gluon}

In order to be able to extract the soft limit factor $S_{m}\left(1^{+}, 2^{+}, \ldots i^{-}, \ldots m^{+}\right)$we consider the NMHV n-gluon amplitude $A_{n}\left(1^{+}, \ldots, i^{-}, \ldots k^{-}, \ldots, l^{-}, \ldots, n\right)$. We would like to stress that the reason for choosing the NMHV n-gluon amplitude is purely for simplicity. As will become obvious from the diagrammatic analysis below, the same universal factorisation (2.2) is valid for any chosen initial amplitude $A_{n}$. The only difference will be that the $A_{n-m}$ amplitude that factorises will have more than 2 negative helicity gluons. From now on we will omit writing explicitly the positive helicity gluons unless needed. As discussed above in this section we will take the simultaneous soft limit of $m$ gluons of which only one will be of negative helicity. Then the multi-soft factor can be identified from the following relation

$$
\begin{aligned}
& \lim _{\delta \rightarrow 0} A_{n}\left(i^{-}, k^{-}, l^{-}\right)=S_{m}\left(1^{+}, 2^{+}, \ldots i^{-}, \ldots m^{+}\right) A_{n-m}\left(m+1, \ldots, k^{-}, \ldots l^{-}, \ldots, n\right)+\mathcal{O}(\delta), \\
& S_{m}\left(1^{+}, 2^{+}, \ldots i^{-}, \ldots m^{+}\right)=\sum_{a=0}^{m} \frac{1}{\delta^{m-a}} S_{m}^{(a)}\left(i^{-}\right) .
\end{aligned}
$$



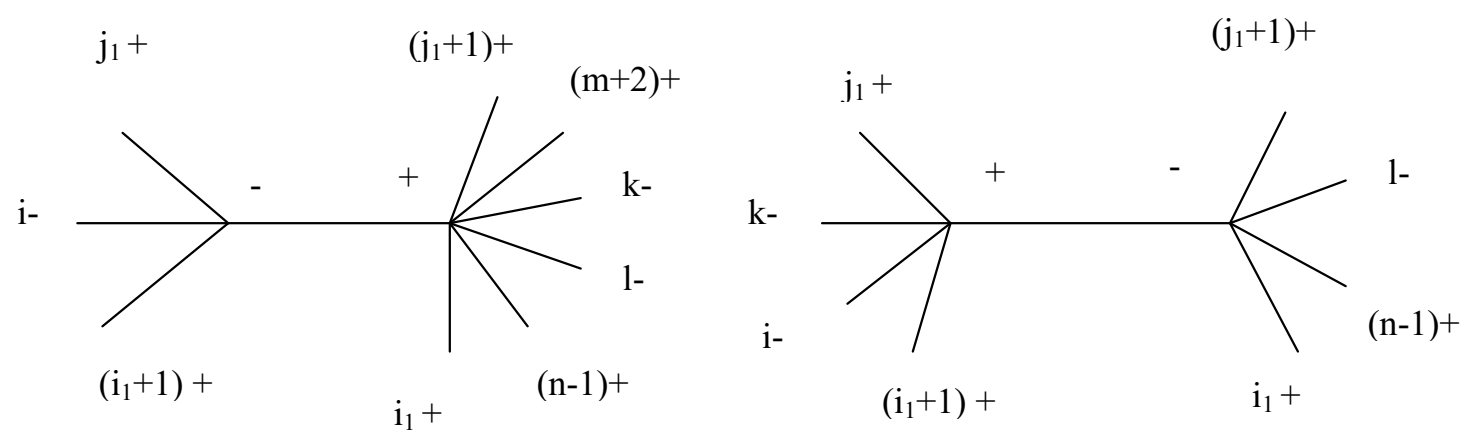

Figure 1. On the left tree diagrams with $\mathrm{MHV}$ vertices contributing to the leading multi-soft factor $S_{m}^{(0)}\left(1^{+}, 2^{+}, \ldots, i^{-}, \ldots m^{+}\right)$. On the right tree diagrams which give subleading contributions to the multi-soft factor. In the case of the factorisation of a generic "hard" amplitude the right vertex should be "dressed" with additional MHV vertices. Apparently, this will not change the soft factor (2.5), (2.11).

In what follows we will concentrate on the leading term $S_{m}^{(0)}$ in the expansion of (2.2). We should mention that there is no obstacle in going beyond the leading order.

Actually, there are two classes of diagrams that can potentially contribute to $S_{m}^{(0)}$. They are depicted in figure 1 . To start with notice that all the diagrams on the right of figure 1 , which have only one negative helicity gluon, either $k$ or $l$ on the right vertex are absent. To convince ourselves lets us count the powers of $\delta$ of such a diagram. The right MHV vertex will behave like $1 / \delta^{i_{1}}$. This scaling comes from the fact the right MHV vertex has $2 i_{1}$ holomorphic spinors in its denominator each of which scales as $\sqrt{\delta}$. Notice that when two or more of the hard gluons appear in the left vertex then the propagator is finite in the multi-soft limit (2.1) and as a consequence it does not contribute any power of $\delta$ in the leading term $S_{m}^{(0)}$ of the soft factor. Similarly, the left MHV vertex behaves like $\delta^{2} / \delta^{m-i_{1}}$, where the additional $\delta^{2}$ in the numerator is coming from the $\langle i k\rangle^{4}$ term present in the numerator of the left MHV vertex. As a result, the whole diagram scales like $1 / \delta^{m-2}$ which gives contribution only in the sub-subleading soft factor $S_{m}^{(2)}$. Actually we will see that this is a generic feature, namely all diagrams with more than two hard particles belonging to the same MHV vertex which has a negative helicity soft gluon will not contribute to the leading and sub-leading soft factors $S_{m}^{(0)}$ and $S_{m}^{(1)}$ and can safely be ignored.

Using the expressions for the gluonic MHV vertices, one can write down an analytic expression for the left diagram of figure 1 :

$$
\begin{aligned}
A_{n}^{(1)}= & \sum_{i_{1}=n-1}^{i-1} \sum_{j_{1}=i}^{\prime} \frac{\left\langle\left(i_{1}+1, j_{1}\right) i\right\rangle^{4}}{\left\langle\left(i_{1}+1, j_{1}\right) i_{1}+1\right\rangle \ldots\left\langle j_{1}-1 j_{1}\right\rangle\left\langle j_{1}\left(i_{1}+1, j_{1}\right)\right\rangle} \frac{1}{P_{i_{1}+1, j_{1}}^{2}} \\
& \times \frac{\langle k l\rangle^{4}}{\left\langle\left(i_{1}+1, j_{1}\right) j_{1}+1\right\rangle \ldots\left\langle i_{1}-1 i_{1}\right\rangle\left\langle i_{1}\left(i_{1}+1, j_{1}\right)\right\rangle},
\end{aligned}
$$

where the primes at the sums are to remind us that we should omit the term for $i_{1}=n-1$ and $j_{1}=m+1$ since this term has two hard gluons on the left MHV vertex and as argued above is subleading in the soft limit. In the last expression the quantity $\left|\left(i_{1}+1, j_{1}\right)\right\rangle$ 
denotes the spinor that corresponds to the off-shell momentum of the propagator $P_{i_{1}+1, j_{1}}$. It is defined as usual $\lambda_{\left(i_{1}+1, j_{1}\right)} \alpha=P_{i_{1}+1, j_{1}} \alpha \dot{\alpha} \zeta^{\dot{\alpha}}$, where $\zeta^{\dot{\alpha}}$ is an arbitrary reference spinor.

Taking the multi-soft limit (2.1) this expression can be easily rewritten as

$$
A_{n}^{(1)}=\frac{\langle n m+1\rangle}{\prod_{q=n}^{m}\langle q q+1\rangle} \sum_{i_{1}=n-1}^{i-1} \sum_{j_{1}=i}^{\prime} \frac{\left\langle i^{-}\left|\not p_{i_{1}+1, j_{1}}\right| \zeta^{-}\right\rangle^{4}}{D} A_{n-m}\left(m+1^{+}, \ldots k^{-}, l^{-}, \ldots, n^{+}\right) .
$$

where we have defined the universal combination,

$$
D=\left\langle i_{1}^{-}\left|\not p_{i_{1}+1, j_{1}}\right| \zeta^{-}\right\rangle\left\langle j_{1}+1^{-}\left|\not p_{i_{1}+1, j_{1}}\right| \zeta^{-}\right\rangle\left\langle i_{1}+1^{-}\left|\not p_{i_{1}+1, j_{1}}\right| \zeta^{-}\right\rangle\left\langle j_{1}^{-}\left|\not p_{i_{1}+1, j_{1}}\right| \zeta^{-}\right\rangle \frac{P_{i_{1}+1, j_{1}}^{2}}{\left\langle i_{1} i_{1}+1\right\rangle\left\langle j_{1} j_{1}+1\right\rangle},
$$

and, as usual, $\zeta$ is the reference spinor of the CSW method.

From (2.3) it is direct to read the multi-soft factor that we are after. It reads

$$
S_{m}^{(0)}\left(i^{-}\right)=\frac{\langle n m+1\rangle}{\prod_{q=n}^{m}\langle q q+1\rangle} \sum_{i_{1}=n-1}^{i-1} \sum_{j_{1}=i}^{m+1} \frac{\left\langle i^{-}\left|\not \phi_{i_{1}+1, j_{1}}\right| \zeta^{-}\right\rangle^{4}}{D} .
$$

A number of important comments are in order. First of all, notice that our result (2.5) appears to depend on the arbitrary reference spinor $\zeta$. We will now argue that the result (2.5) is actually independent of the reference spinor $\zeta$. On one hand, we know that the full amplitude is independent of the value of the reference spinor [50]. As a result, any multi-soft limiting process should give a result that should be also independent of the reference spinor. In fact, each term $S_{m}^{(a)}\left(i^{-}\right)$in the $1 / \delta$ expansion of $S_{m}\left(i^{-}\right)(2.2)$ should be separately independent since the cancellations are only possible between terms that have the same power divergence $1 / \delta^{a}$ in the multi-soft limit. We conclude that the soft factor we have calculated and which is the leading singularity of the full amplitude should not depend on which reference spinor one chooses. Below we will verify this for the simple case of the double-soft limit. In practice, we will choose $\zeta$ to be the anti-holomorphic spinor of a positive helicity gluon, namely $\zeta^{\dot{\alpha}}=\tilde{\lambda}_{n}^{\dot{\alpha}}$ or $\zeta^{\dot{\alpha}}=\tilde{\lambda}_{1}^{\dot{\alpha}}$. As a consequence, our result will be Lorentz and gauge invariant and free of singularities connected to the choice of the reference spinor. A second comment concerns the structure of our result (2.5). Notice that this result is the expression for the multi-soft limit of $m$ adjacent positive helicity gluons multiplied by a correction term given by the double sum.

The careful reader might worry that the expression (2.5) depends not only on the momenta of the soft gluons $g_{1}, \ldots, g_{m}$ and their adjacent particles $n$ and $m+1$, as it should, but also on the momenta of the next-to-adjacent gluons, i.e. $n-1$ and $m+2$ since the sums (2.5) involve these gluons. To clarify this point, one should evaluate the boundary terms of (2.2), that is the terms for $i_{1}=n-1$ or $j_{1}=m+1$. By doing so it is easy to see that the dependence on the $n-1$ and $m+2$ gluons drops out. Indeed, for $i_{1}=n-1$ the double sum of (2.5) simplifies to

$$
S_{m}^{\left(i_{1}=n-1\right)}\left(i^{-}\right)=\frac{\langle n m+1\rangle}{\prod_{q=n}^{m}\langle q q+1\rangle} \sum_{j_{1}=i}^{m} \frac{\left\langle i^{-}\left|\not p_{n}\right| \zeta^{-}\right\rangle^{4}}{D}
$$


with the denominator being

$$
D=[n \zeta]\left\langle j_{1}+1^{-}\left|\not \phi_{n}\right| \zeta^{-}\right\rangle\left\langle n^{-}\left|\phi_{1, j_{1}}\right| \zeta^{-}\right\rangle\left\langle j_{1}^{-}\left|\not p_{n}\right| \zeta^{-}\right\rangle \frac{2 p_{n} \cdot q_{1, j_{1}}}{\left\langle j_{1} j_{1}+1\right\rangle} .
$$

Thus the last two expressions are explicitly independent of the gluon $n-1$.

Similarly, for the other boundary term $j_{1}=m+1$ we get

$$
S_{m}^{\left(j_{1}=m+1\right)}\left(i^{-}\right)=\frac{\langle n m+1\rangle}{\prod_{q=n}^{m}\langle q q+1\rangle} \sum_{i_{1}=n}^{i-1} \frac{\left\langle i^{-}\left|\not \phi_{m+1}\right| \zeta^{-}\right\rangle^{4}}{D},
$$

with the denominator being

$$
D=-\left\langle i_{1}^{-}\left|\not p_{m+1}\right| \zeta^{-}\right\rangle[m+1 \zeta]\left\langle i_{1}+1^{-}\left|\not p_{m+1}\right| \zeta^{-}\right\rangle\left\langle m+1^{-}\left|\not p_{i_{1}+1, m}\right| \zeta^{-}\right\rangle \frac{2 p_{m+1} \cdot q_{i_{1}+1, m}}{\left\langle i_{1} i_{1}+1\right\rangle}
$$

which gives a result independent of the details of the $m+2$ gluon.

One can use the general expression (2.5) to obtain the double soft factor when the gluons $1^{+}$and $2^{-}$become soft simultaneously. There are four terms which after a bit of algebra read

$$
\begin{aligned}
S_{2}^{(a)}\left(1^{+}, 2^{-}\right) & =\frac{\langle n 3\rangle}{\langle 12\rangle q_{12}^{2}} \frac{\left\langle 2^{-}\left|\not p_{1}\right| \zeta^{-}\right\rangle^{3}}{\left\langle 1^{-}\left|p_{2}\right| \zeta^{-}\right\rangle\left\langle 3^{-}\left|\phi_{12}\right| \zeta^{-}\right\rangle\left\langle n^{-}\left|q_{12}\right| \zeta^{-}\right\rangle} \\
S_{2}^{(b)}\left(1^{+}, 2^{-}\right) & =-\frac{\langle n 3\rangle}{\langle 12\rangle\langle 23\rangle 2 q_{12} \cdot p_{3}} \frac{\left\langle 2^{-}\left|p_{3}\right| \zeta^{-}\right\rangle^{4}}{\left\langle 1^{-}\left|p_{3}\right| \zeta^{-}\right\rangle\left\langle 3^{-}\left|\phi_{12}\right| \zeta^{-}\right\rangle\left\langle n^{-}\left|\not p_{3}\right| \zeta^{-}\right\rangle[3 \zeta]} \\
S_{2}^{(c)}\left(1^{+}, 2^{-}\right) & =-\frac{\langle n 3\rangle}{\langle n 1\rangle\langle 23\rangle 2 q_{2} \cdot p_{3}} \frac{\left\langle 2^{-}\left|p_{3}\right| \zeta^{-}\right\rangle^{3}}{\left\langle 3^{-}\left|p_{2}\right| \zeta^{-}\right\rangle\left\langle 1^{-}\left|\not p_{3}\right| \zeta^{-}\right\rangle[3 \zeta]} \\
S_{2}^{(d)}\left(1^{+}, 2^{-}\right) & =\frac{\langle n 3\rangle}{\langle 12\rangle\langle n 1\rangle 2 q_{12} \cdot p_{n}} \frac{\left\langle 2^{-}\left|p_{n}\right| \zeta^{-}\right\rangle^{3}}{\left\langle 3^{-}\left|\not p_{n}\right| \zeta^{-}\right\rangle\left\langle n^{-}\left|q_{12}\right| \zeta^{-}\right\rangle[n \zeta]}
\end{aligned}
$$

We have checked numerically that the sum of the four terms above is indeed independent of the reference spinor $\zeta^{\dot{\alpha}}$.

It is convenient to choose as the reference spinor that of first positive helicity gluon $1^{+}$, that is $\zeta^{\dot{\alpha}}=\tilde{\lambda}_{1}^{\dot{\alpha}}$. Then the first contribution $S_{2}^{(a)}\left(1^{+}, 2^{-}\right)=0$ vanishes and the final result is a sum of the last three terms in (2.10) after the substitution $\zeta^{\dot{\alpha}}=\tilde{\lambda}_{1}^{\dot{\alpha}}$. After some simple algebra we get the final result

$$
S_{2}\left(1^{+}, 2^{-}\right)=\frac{1}{\langle 12\rangle[21]}\left(\frac{\langle 23\rangle^{2}[31]}{\langle 13\rangle 2 q_{12} \cdot p_{3}}+\frac{\langle 12\rangle\langle 23\rangle\langle n 3\rangle[31]}{\langle n 1\rangle\langle 13\rangle 2 q_{2} \cdot p_{3}}+\frac{\langle 2 n\rangle^{2}[n 1]}{\langle n 1\rangle 2 q_{12} \cdot p_{n}}\right) .
$$

One can check that this result is another representation of the double soft limit

$$
S_{2}\left(1^{+}, 2^{-}\right)=\frac{1}{\left\langle n^{-}\left|q_{12}\right| 3^{-}\right\rangle}\left(\frac{1}{2 p_{n} \cdot q_{12}} \frac{\langle n 2\rangle^{3}[n 3]}{\langle n 1\rangle\langle 12\rangle}-\frac{1}{2 p_{3} \cdot q_{12}} \frac{\langle n 3\rangle[31]^{3}}{[12][23]}\right)
$$

first obtained in $[24,25]$.

At this point we wish to comment on the effectiveness of the CSW method compared to this of the BCFW method. First of all from the CSW method we directly get final 
expression for the multi-soft factor and not recursive relations. Secondly, if we roughly estimate the number of terms in the sums of the CSW method this will be the number of diagrams with different topology times $m^{2 n}$, where $m$ is the number of soft particles and $n$ the number of negative helicity gluons since each negative helicity gluon brings in a double sum (see (2.5) and the results of the next sections). On the other hand, due to the recursive nature of the $\mathrm{BCFW}$ method in order to calculate the $m^{\text {th }}$ multi-soft limit one has a sum of $m-1$ terms corresponding to all multi-soft factors from $S_{2}$ to $S_{m-1}$. Now each of these multi-soft factors is written as a sum of all the lower point ones and so on. As a result, the number of terms in the final result of the BCFW method grows exponentially $\left(\sim 2^{m}\right)$ with respect to the number of soft particles. Thus for $m \gg 1$ and $n \ll m / 2$ the number of terms in the CSW result is much smaller than the one in the BCFW method.

\subsection{Rules for generic gluonic multi-soft factor}

In this section, we show how to construct a generic multi-soft factor $S_{m}\left(\left(i_{1}\right)^{-},\left(i_{2}\right)^{-}, \ldots,\left(i_{k}\right)^{-}\right)$with the aid of MHV diagrams. The limit we take is the simultaneous soft limit of $m$ gluons $k$ of which are of negative helicity. Having the experience of the simple example in the previous section it is not difficult to write down the general rules for such a multi-soft limit. We should stress that these rules are independent of which "hard" amplitude factorises and multiplies the multi-soft factor $S_{m}$ when the multi-soft limit is taken. As a result the multi-soft factorisation is universal with the amplitude that factorises being independent of the momenta of the soft gluons and with the multi-soft factor $S_{m}$ depending only on the momenta of the soft particles, as well as on the two hard gluons which are adjacent to the soft ones, i.e. the $n$ and $m+1$ gluons.

1. Draw all different topologically different "skeleton" MHV diagrams each with $k-1$ MHV vertices.

2. Distribute the negative helicity gluons among the MHV vertices of each "skeleton" diagram in all inequivalent ways. Then distribute all positive helicity gluons in all possible ways respecting the ordering of the particles. The particles which one needs to distribute are the soft ones plus the four hard gluons adjacent to the $\mathrm{m}$ soft ones, that is $n, n-1, m+1, m+2$. In accordance with the discussion in the previous section, care should be taken so that the gluons $m+2$ and $n-1$ belong to the same MHV vertex. Otherwise the diagram will give only subleading contribution to the multi-soft factor. ${ }^{4}$

3. For each MHV vertex, except the one which has the $n-1$ and $m+2$ gluons, include a factor of $\left\langle n_{1} n_{2}\right\rangle^{4}$ in the numerator, where $n_{1}$ and $n_{2}$ are the negative helicity gluons (internal or external) of the MHV vertex.

4. For each propagator of momentum $P_{i}$ connecting two MHV vertices include a factor $1 / D_{i}$, where

$$
D_{i}=\left\langle j_{1}^{-}\left|\not p_{i}\right| \zeta^{-}\right\rangle\left\langle j_{3}^{-}\left|\not p_{i}\right| \zeta^{-}\right\rangle\left\langle j_{4}^{-}\left|\not p_{i}\right| \zeta^{-}\right\rangle\left\langle j_{2}^{-}\left|\not p_{i}\right| \zeta^{-}\right\rangle P_{i}^{2}
$$

\footnotetext{
${ }^{4}$ As discussed in the previous section the dependence on the gluons $m+2$ and $n-1$ drops out of the final result.
} 
$\left(\mathrm{a}_{1}\right)$

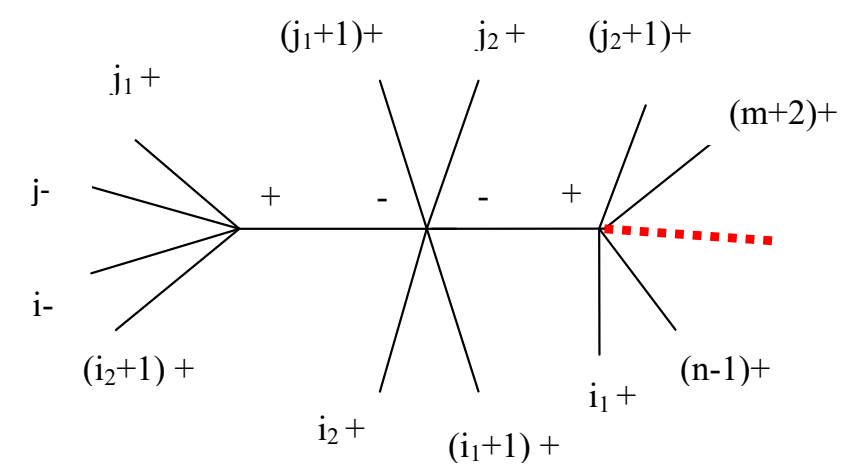

$$
\left(\mathrm{j}_{1}+1\right)+\quad \mathrm{j}_{2}+\quad\left(\mathrm{j}_{2}+1\right)+
$$

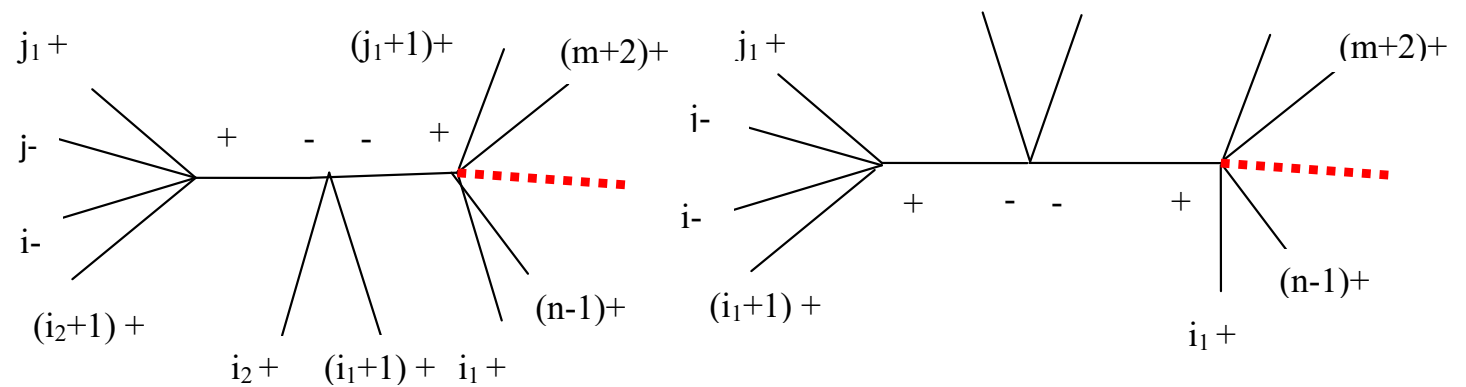

$\left(a_{3}\right)$

$\left(a_{2}\right)$

Figure 2. Diagrams contributing to the multi-soft factor involving 2 negative helicity gluons $S_{m}^{(0)}\left(1^{+}, \ldots, i^{-}, \ldots, j^{-}, \ldots, m^{+}\right)$. The negative helicity gluons are on the left MHV vertex. The red dashed line denotes the additional gluons and possibly vertices of the hard amplitude that factorises in the soft limit.

where $j_{1}, j_{2}, j_{3}, j_{4}$ are the four gluons adjacent to the propagator under consideration. Notice that it might happen that one or more of these gluons are propagators too. If a diagram has propagators which are adjacent multiply by $\left\langle P_{i} P_{j}\right\rangle=-\left\langle\zeta^{+}\left|\not p_{i} \not \phi_{j}\right| \zeta^{-}\right\rangle$ for each set of adjacent propagators $\left(P_{i}, P_{j}\right)$.

5. Multiply by a factor $\left\langle j_{1} j_{1}+1\right\rangle$ for all adjacent external particles which do not belong to the same MHV vertex.

6. Finally, sum over the contributions of all diagrams and multiply by the ubiquitous factor $U=\frac{\langle n m+1\rangle}{\prod_{q=n}^{m}\langle q q+1\rangle}$.

\subsection{Gluonic multi-soft factor with two negative helicity gluons}

In this section, as an example of the aforementioned rules we give compact expressions for the leading singularity of the gluonic multi-soft factor with two negative and $m-2$ positive helicity gluons. In this section, our choice for the reference spinor will be $\zeta^{\dot{\alpha}}=\lambda_{n}^{\dot{\alpha}}$. 
There is a total of nine topologically different diagrams which contribute to this multisoft limit. They are depicted in figures 2, 3 and 4 . For the fist diagram (a1) of figure 2 we get

$$
\begin{array}{r}
S_{m}^{\left(a_{1}\right)}\left(i^{-}, j^{-}\right)=U \sum_{i_{1}=n-1}^{i-2} \sum_{i_{2}=i_{1}+1}^{\prime} \sum_{j_{1}=j} \sum_{j_{2}=j_{1}+1}^{m+1} \frac{\langle i j\rangle^{4}\left\langle n^{+}\left|\not p_{i_{1}+1, j_{2}} \not \phi_{i_{2}+1, j_{1}}\right| n^{-}\right\rangle^{4}}{D_{1} D_{2}} \\
\times\left\langle i_{1} i_{1}+1\right\rangle\left\langle i_{2} i_{2}+1\right\rangle\left\langle j_{1} j_{1}+1\right\rangle\left\langle j_{2} j_{2}+1\right\rangle,
\end{array}
$$

where

$$
\begin{aligned}
& D_{1}=\left\langle i_{2}^{-}\left|\not \phi_{i_{2}+1, j_{1}}\right| n^{-}\right\rangle\left\langle i_{2}+1^{-}\left|\not p_{i_{2}+1, j_{1}}\right| n^{-}\right\rangle\left\langle j_{1}^{-}\left|\not p_{i_{2}+1, j_{1}}\right| n^{-}\right\rangle\left\langle j_{1}+1^{-}\left|\not p_{i_{2}+1, j_{1}}\right| n^{-}\right\rangle P_{i_{2}+1, j_{1}}^{2}, \\
& D_{2}=\left\langle i_{1}^{-}\left|\not \phi_{i_{1}+1, j_{2}}\right| n^{-}\right\rangle\left\langle i_{1}+1^{-}\left|\not p_{i_{1}+1, j_{2}}\right| n^{-}\right\rangle\left\langle j_{2}+1^{-}\left|\not p_{i_{1}+1, j_{2}}\right| n^{-}\right\rangle\left\langle j_{2}^{-}\left|\not \phi_{i_{1}+1, j_{2}}\right| n^{-}\right\rangle P_{i_{1}+1, j_{2}}^{2}
\end{aligned}
$$

The other two diagrams (a2) and (a3) of figure 2 yield

$$
\begin{array}{r}
S_{m}^{\left(a_{2}\right)}\left(i^{-}, j^{-}\right)=U \sum_{i_{1}=n-1}^{i-1} \sum_{j_{1}=j}^{\prime} \sum_{j_{2}=j_{1}+1}^{m+1} \frac{\langle i j\rangle^{4}\left\langle n^{+}\left|\not \phi_{i_{1}+1, j_{2}} \not \phi_{i_{1}+1, j_{1}}\right| n^{-}\right\rangle^{3}}{D_{1} D_{2}} \\
\times\left\langle i_{1} i_{1}+1\right\rangle\left\langle j_{1} j_{1}+1\right\rangle\left\langle j_{2} j_{2}+1\right\rangle,
\end{array}
$$

where

$$
\begin{aligned}
& D_{1}=\left\langle i_{1}+1^{-}\left|\not \phi_{i_{1}+1, j_{1}}\right| n^{-}\right\rangle\left\langle j_{1}^{-}\left|\not \phi_{i_{1}+1, j_{1}}\right| n^{-}\right\rangle\left\langle j_{1}+1^{-}\left|\not p_{i_{1}+1, j_{1}}\right| n^{-}\right\rangle P_{i_{1}+1, j_{1}}^{2}, \\
& D_{2}=\left\langle i_{1}^{-}\left|\not \phi_{i_{1}+1, j_{2}}\right| n^{-}\right\rangle\left\langle j_{2}+1^{-}\left|\not \phi_{i_{1}+1, j_{2}}\right| n^{-}\right\rangle\left\langle j_{2}^{-}\left|\not \phi_{i_{1}+1, j_{2}}\right| n^{-}\right\rangle P_{i_{1}+1, j_{2}}^{2} \\
& S_{m}^{\left(a_{3}\right)}\left(i^{-}, j^{-}\right)=-U \sum_{i_{1}=n-1}^{i-2} \sum_{i_{2}=i_{1}+1}^{i-1} \sum_{j_{1}=j}^{\prime m+1} \frac{\langle i j\rangle^{4}\left\langle n^{+}\left|\not p_{i_{1}+1, j_{1}} \not p_{i_{2}+1, j_{1}}\right| n^{-}\right\rangle^{3}}{D_{1} D_{2}} \\
& \times\left\langle i_{1} i_{1}+1\right\rangle\left\langle j_{1} j_{1}+1\right\rangle\left\langle i_{2} i_{2}+1\right\rangle,
\end{aligned}
$$

where

$$
\begin{aligned}
& D_{1}=\left\langle i_{2}^{-}\left|\not p_{i_{2}+1, j_{1}}\right| n^{-}\right\rangle\left\langle j_{1}^{-}\left|\not \phi_{i_{2}+1, j_{1}}\right| n^{-}\right\rangle\left\langle i_{2}+1^{-}\left|\not \phi_{i_{2}+1, j_{1}}\right| n^{-}\right\rangle P_{i_{2}+1, j_{1}}^{2}, \\
& D_{2}=\left\langle i_{1}^{-}\left|\not p_{i_{1}+1, j_{1}}\right| n^{-}\right\rangle\left\langle j_{1}+1^{-}\left|\not \phi_{i_{1}+1, j_{1}}\right| n^{-}\right\rangle\left\langle i_{1}+1^{-}\left|\not \phi_{i_{1}+1, j_{1}}\right| n^{-}\right\rangle P_{i_{1}+1, j_{1}}^{2}
\end{aligned}
$$

We now proceed to evaluate the four diagrams of figure 3 . These give

$$
\begin{array}{r}
S_{m}^{\left(b_{1}\right)}\left(i^{-}, j^{-}\right)=U \sum_{i_{1}=n-1}^{i-2} \sum_{i_{2}=i_{1}+1} \sum_{\substack{j_{1}=i \\
j_{2}}} \sum_{j_{2}=j}^{j-1} \frac{\left\langle i^{-}\left|\not p_{i_{2}+1, j_{1}}\right| n^{-}\right\rangle^{4}\left\langle j^{-}\left|\not p_{i_{1}+1, j_{2}}\right| n^{-}\right\rangle^{4}}{D_{1} D_{2}} \\
\quad<\left\langle i_{1} i_{1}+1\right\rangle\left\langle i_{2} i_{2}+1\right\rangle\left\langle j_{1} j_{1}+1\right\rangle\left\langle j_{2} j_{2}+1\right\rangle,
\end{array}
$$

where

$$
\begin{aligned}
& D_{1}=\left\langle i_{2}^{-}\left|\not p_{i_{2}+1, j_{1}}\right| n^{-}\right\rangle\left\langle i_{2}+1^{-}\left|\not p_{i_{2}+1, j_{1}}\right| n^{-}\right\rangle\left\langle j_{1}^{-}\left|\not p_{i_{2}+1, j_{1}}\right| n^{-}\right\rangle\left\langle j_{1}+1^{-}\left|\not p_{i_{2}+1, j_{1}}\right| n^{-}\right\rangle P_{i_{2}+1, j_{1}}^{2}, \\
& D_{2}=\left\langle i_{1}^{-}\left|\not p_{i_{1}+1, j_{2}}\right| n^{-}\right\rangle\left\langle i_{1}+1^{-}\left|\not p_{i_{1}+1, j_{2}}\right| n^{-}\right\rangle\left\langle j_{2}+1^{-}\left|\not p_{i_{1}+1, j_{2}}\right| n^{-}\right\rangle\left\langle j_{2}^{-}\left|\not p_{i_{1}+1, j_{2}}\right| n^{-}\right\rangle P_{i_{1}+1, j_{2}}^{2}
\end{aligned}
$$


$\left(b_{3}\right)$
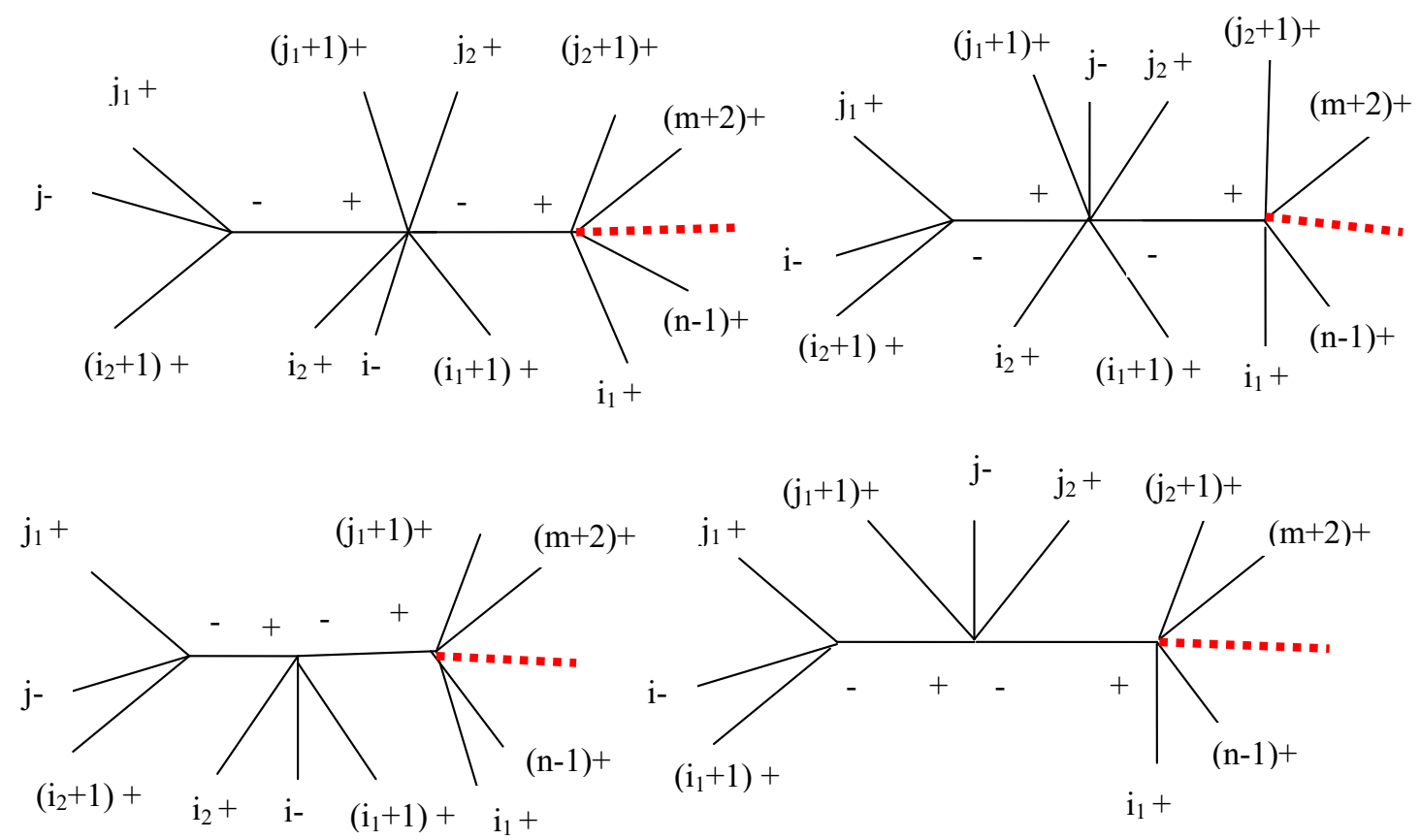

$\left(b_{4}\right)$

Figure 3. Diagrams contributing to the multi-soft factor involving 2 negative helicity gluons $S_{m}^{(0)}\left(1^{+}, \ldots, i^{-}, \ldots, j^{-}, \ldots, m^{+}\right)$. Here the negative helicity gluons are sitting one on the left MHV vertex and one on the middle MHV vertex. The red dashed line denotes the additional gluons and possibly vertices of the hard amplitude that factorises in the soft limit.

$$
\begin{aligned}
S_{m}^{\left(b_{2}\right)}\left(i^{-}, j^{-}\right)=U \sum_{i_{1}=n-1}^{i-1} \sum_{j_{1}=i}^{\prime} \sum_{j_{2}=j}^{j-1}{ }^{\prime} \frac{\left\langle i^{-}\left|\not \phi_{i_{1}+1, j_{1}}\right| n^{-}\right\rangle^{4}\left\langle j^{-}\left|\not \phi_{i_{1}+1, j_{2}}\right| n^{-}\right\rangle^{4}}{D_{1} D_{2}} \\
\times\left\langle i_{1} i_{1}+1\right\rangle\left\langle j_{1} j_{1}+1\right\rangle\left\langle j_{2} j_{2}+1\right\rangle,
\end{aligned}
$$

where

$$
\begin{gathered}
D_{1}=\left\langle i_{1}+1^{-}\left|\not p_{i_{1}+1, j_{1}}\right| n^{-}\right\rangle\left\langle j_{1}^{-}\left|\not p_{i_{1}+1, j_{1}}\right| n^{-}\right\rangle\left\langle j_{1}+1^{-}\left|\not p_{i_{1}+1, j_{1}}\right| n^{-}\right\rangle P_{i_{1}+1, j_{1}}^{2}, \\
D_{2}=\left\langle i_{1}^{-}\left|\not p_{i_{1}+1, j_{2}}\right| n^{-}\right\rangle\left\langle n^{+}\left|\not p_{i_{1}+1, j_{2}} \not p_{i_{1}+1, j_{1}}\right| n^{-}\right\rangle\left\langle j_{2}+1^{-}\left|\not p_{i_{1}+1, j_{2}}\right| n^{-}\right\rangle\left\langle j_{2}^{-}\left|\not p_{i_{1}+1, j_{2}}\right| n^{-}\right\rangle P_{i_{1}+1, j_{2}}^{2} \\
S_{m}^{\left(b_{3}\right)}\left(i^{-}, j^{-}\right)=U \sum_{i_{1}=n-1}^{i-1^{\prime}} \sum_{i_{2}=i}^{j-1} \sum_{j_{1}=j}^{\prime m} \sum_{j_{2}=j_{1}+1}^{m+1} \frac{\left\langle j^{-}\left|\not p_{i_{2}+1, j_{1}}\right| n^{-}\right\rangle^{4}\left\langle i^{-}\left|\not p_{i_{1}+1, j_{2}}\right| n^{-}\right\rangle^{4}}{D_{1} D_{2}} \\
\times\left\langle i_{1} i_{1}+1\right\rangle\left\langle i_{2} i_{2}+1\right\rangle\left\langle j_{1} j_{1}+1\right\rangle\left\langle j_{2} j_{2}+1\right\rangle,
\end{gathered}
$$




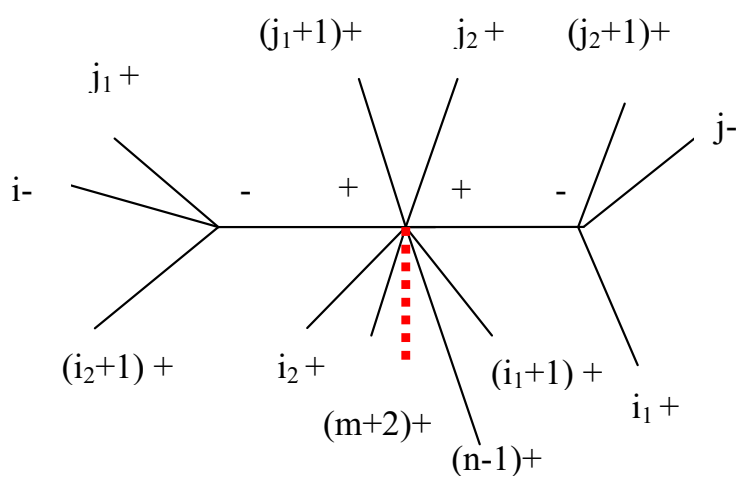

$\left(c_{1}\right)$

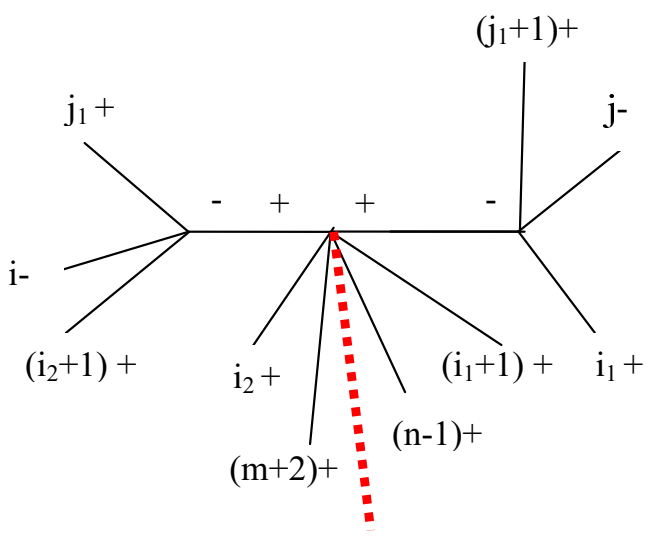

(c)

Figure 4. Diagrams contributing to the multi-soft factor involving 2 negative helicity gluons $S_{m}^{(0)}\left(1^{+}, \ldots, i^{-}, \ldots, j^{-}, \ldots, m^{+}\right)$. Here the negative helicity gluons are sitting one on the left MHV vertex and one on the right MHV vertex. The red dashed line denotes the additional gluons and possibly vertices of the hard amplitude that factorises in the soft limit.

where

$$
\begin{aligned}
& D_{1}=\left\langle i_{2}^{-}\left|\not \phi_{i_{2}+1, j_{1}}\right| n^{-}\right\rangle\left\langle i_{2}+1^{-}\left|\not \phi_{i_{2}+1, j_{1}}\right| n^{-}\right\rangle\left\langle j_{1}^{-}\left|\not p_{i_{2}+1, j_{1}}\right| n^{-}\right\rangle\left\langle j_{1}+1^{-}\left|\not p_{i_{2}+1, j_{1}}\right| n^{-}\right\rangle P_{i_{2}+1, j_{1}}^{2}, \\
& D_{2}=\left\langle i_{1}^{-}\left|\not \phi_{i_{1}+1, j_{2}}\right| n^{-}\right\rangle\left\langle i_{1}+1^{-}\left|\not \phi_{i_{1}+1, j_{2}}\right| n^{-}\right\rangle\left\langle j_{2}+1^{-}\left|\not p_{i_{1}+1, j_{2}}\right| n^{-}\right\rangle\left\langle j_{2}^{-}\left|\not p_{i_{1}+1, j_{2}}\right| n^{-}\right\rangle P_{i_{1}+1, j_{2}}^{2}
\end{aligned}
$$

$$
\begin{array}{r}
S_{m}^{\left(b_{4}\right)}\left(i^{-}, j^{-}\right)=-U \sum_{i_{1}=n-1}^{i-1} \sum_{i_{2}=i}^{\prime} \sum_{j_{1}=j}^{j-1}{ }^{\prime m+1} \frac{\left\langle j^{-}\left|\not p_{i_{2}+1, j_{1}}\right| n^{-}\right\rangle^{4}\left\langle i^{-}\left|\not p_{i_{1}+1, j_{1}}\right| n^{-}\right\rangle^{4}}{D_{1} D_{2}} \\
\times\left\langle i_{1} i_{1}+1\right\rangle\left\langle i_{2} i_{2}+1\right\rangle\left\langle j_{1} j_{1}+1\right\rangle,
\end{array}
$$

where

$$
\begin{aligned}
& D_{1}=\left\langle i_{2}^{-}\left|\not p_{i_{2}+1, j_{1}}\right| n^{-}\right\rangle\left\langle i_{2}+1^{-}\left|\not p_{i_{2}+1, j_{1}}\right| n^{-}\right\rangle\left\langle j_{1}^{-}\left|\not p_{i_{2}+1, j_{1}}\right| n^{-}\right\rangle P_{i_{2}+1, j_{1}}^{2}, \\
& D_{2}=\left\langle i_{1}^{-}\left|\not p_{i_{1}+1, j_{1}}\right| n^{-}\right\rangle\left\langle i_{1}+1^{-}\left|\not p_{i_{1}+1, j_{1}}\right| n^{-}\right\rangle\left\langle j_{1}+1^{-}\left|\not p_{i_{1}+1, j_{1}}\right| n^{-}\right\rangle\left\langle n^{+}\left|\not p_{i_{2}+1, j_{1}} \not p_{i_{1}+1, j_{1}}\right| n^{-}\right\rangle P_{i_{1}+1, j_{1}}^{2}
\end{aligned}
$$

Finally, from the diagrams of figure 3 one gets

$$
\begin{array}{r}
S_{m}^{\left(c_{1}\right)}\left(i^{-}, j^{-}\right)=U \sum_{i_{1}=j}^{n-1} \sum_{i_{2}=m+2} \sum_{\begin{array}{l}
j_{1}=i \\
j_{2}
\end{array}} \sum_{j_{2}=j_{1}+1}^{j-1} \frac{\left\langle j^{-}\left|\not p_{j_{2}+1, i_{1}}\right| n^{-}\right\rangle^{4}\left\langle i^{-}\left|\not p_{i_{2}+1, j_{1}}\right| n^{-}\right\rangle^{4}}{D_{1} D_{2}} \\
\quad \times\left\langle i_{1} i_{1}+1\right\rangle\left\langle i_{2} i_{2}+1\right\rangle\left\langle j_{1} j_{1}+1\right\rangle\left\langle j_{2} j_{2}+1\right\rangle,
\end{array}
$$




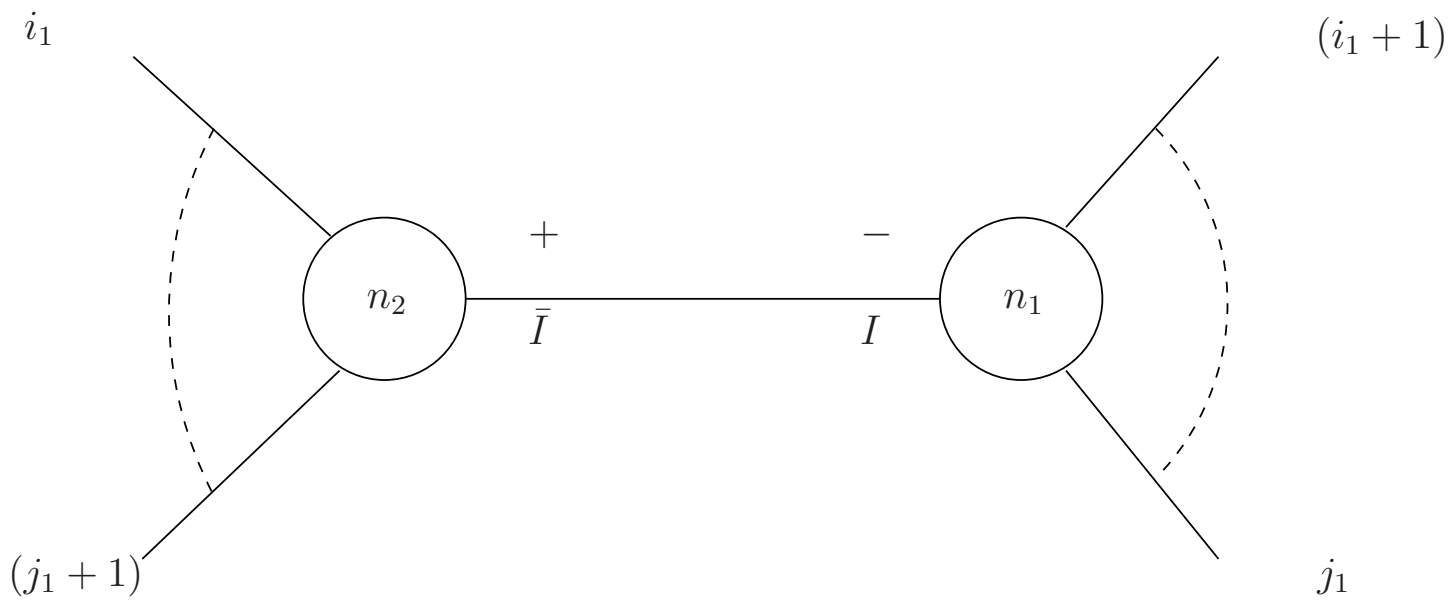

Figure 5. Tree diagrams with 2 analytic supervertices contributing to the degree- 12 superamplitude of eq. (3.7). $n_{1}$ and $n_{2}$ are the number of legs in the right and left vertex respectively.

where

$$
\begin{gathered}
D_{1}=\left\langle i_{2}^{-}\left|\not p_{i_{2}+1, j_{1}}\right| n^{-}\right\rangle\left\langle i_{2}+1^{-}\left|\not p_{i_{2}+1, j_{1}}\right| n^{-}\right\rangle\left\langle j_{1}^{-}\left|\not p_{i_{2}+1, j_{1}}\right| n^{-}\right\rangle\left\langle j_{1}+1^{-}\left|\not p_{i_{2}+1, j_{1}}\right| n^{-}\right\rangle P_{i_{2}+1, j_{1}}^{2}, \\
D_{2}=\left\langle i_{1}^{-}\left|\not p_{j_{2}+1, i_{1}}\right| n^{-}\right\rangle\left\langle i_{1}+1^{-}\left|\not p_{j_{2}+1, i_{1}}\right| n^{-}\right\rangle\left\langle j_{2}+1^{-}\left|\not p_{j_{2}+1, i_{1}}\right| n^{-}\right\rangle\left\langle j_{2}^{-}\left|\not p_{j_{2}+1, i_{1}}\right| n^{-}\right\rangle P_{j_{2}+1, i_{1}}^{2} \\
S_{m}^{\left(c_{2}\right)}\left(i^{-}, j^{-}\right)=-U \sum_{i_{1}=j}^{n-1} \sum_{i_{2}=m+2}^{\prime i^{\prime-1}{ }^{\prime}} \sum_{j_{1}=i}^{j-1} \frac{\left\langle j^{-}\left|\not p_{j_{1}+1, i_{1}}\right| n^{-}\right\rangle^{4}\left\langle i^{-}\left|\not p_{i_{2}+1, j_{1}}\right| n^{-}\right\rangle^{4}}{D_{1} D_{2}} \\
\times\left\langle i_{1} i_{1}+1\right\rangle\left\langle i_{2} i_{2}+1\right\rangle\left\langle j_{1} j_{1}+1\right\rangle,
\end{gathered}
$$

where

$$
\begin{aligned}
& D_{1}=\left\langle i_{2}^{-}\left|\not p_{i_{2}+1, j_{1}}\right| n^{-}\right\rangle\left\langle i_{2}+1^{-}\left|\not \phi_{i_{2}+1, j_{1}}\right| n^{-}\right\rangle\left\langle j_{1}^{-}\left|\not p_{i_{2}+1, j_{1}}\right| n^{-}\right\rangle P_{i_{2}+1, j_{1}}^{2}, \\
& D_{2}=\left\langle i_{1}^{-}\left|\not p_{j_{1}+1, i_{1}}\right| n^{-}\right\rangle\left\langle i_{1}+1^{-}\left|\not p_{j_{1}+1, i_{1}}\right| n^{-}\right\rangle\left\langle j_{1}+1^{-}\left|\not p_{j_{1}+1, i_{1}}\right| n^{-}\right\rangle\left\langle n^{+}\left|\not p_{j_{1}+1, i_{1}} \not p_{i_{2}+1, j_{1}}\right| n^{-}\right\rangle P_{j_{1}+1, i_{1}}^{2}
\end{aligned}
$$

Overall the final result for the multi-soft factor with two negative helicity gluons reads $S_{m}\left(i^{-}, j^{-}\right)=S_{m}^{\left(a_{1}\right)}\left(i^{-}, j^{-}\right)+S_{m}^{\left(a_{2}\right)}\left(i^{-}, j^{-}\right)+S_{m}^{\left(a_{3}\right)}\left(i^{-}, j^{-}\right)+S_{m}^{\left(b_{1}\right)}\left(i^{-}, j^{-}\right)+S_{m}^{\left(b_{2}\right)}\left(i^{-}, j^{-}\right)+$ $S_{m}^{\left(b_{3}\right)}\left(i^{-}, j^{-}\right)+S_{m}^{\left(b_{4}\right)}\left(i^{-}, j^{-}\right)+S_{m}^{\left(c_{1}\right)}\left(i^{-}, j^{-}\right)+S_{m}^{\left(c_{2}\right)}\left(i^{-}, j^{-}\right)$.

\section{Multi-soft limits in $N=4 \mathrm{SYM}$ from the analytic supervertex}

\subsection{Iterating the analytic supervertex}

In this section, we briefly review the application of the CSW method in $\mathcal{N}=4$ SYM following closely [53]. The building block of this construction will be the analytic supervertex of Nair [64].

It is well-known that all analytic amplitudes in generic $0 \leq \mathcal{N} \leq 4$ gauge theories can be combined into a single $\mathcal{N}=4$ supersymmetric expression given by [64],

$$
A_{n}^{\mathcal{N}=4}=\delta^{(8)}\left(\sum_{i=1}^{n} \lambda_{i a} \eta_{i}^{A}\right) \frac{1}{\prod_{i=1}^{n}\langle i i+1\rangle} .
$$


Here $\eta_{i}^{A}$ are anticommuting variables and $A=1,2,3,4$ is an R-symmetry SU(4) index. The Grassmann-valued delta function is defined as follows,

$$
\delta^{(8)}\left(\sum_{i=1}^{n} \lambda_{i a} \eta_{i}^{A}\right) \equiv \prod_{A=1}^{4} \frac{1}{2}\left(\sum_{i=1}^{n} \lambda_{i}^{a} \eta_{i}^{A}\right)\left(\sum_{i=1}^{n} \lambda_{i a} \eta_{i}^{A}\right) .
$$

By Taylor expanding (3.1) in powers of $\eta_{i}$, one can identify each term in the expansion with a particular tree-level analytic amplitude in the $\mathcal{N}=4$ theory. Each factor of $\left(\eta_{i}\right)^{k}$ for $k=0, \ldots, 4$ is interpreted as the $i^{\text {th }}$ particle with helicity $h_{i}=1-\frac{k}{2}$. This implies that helicities take values, $\left\{1, \frac{1}{2}, 0,-\frac{1}{2},-1\right\}$, which precisely correspond to the particles of the $\mathcal{N}=4$ supermultiplet, $\left\{g^{-}, \Lambda_{A}^{-}, \phi^{A B}, \Lambda^{A+}, g^{+}\right\}$.

Actually, it is straightforward to associate a single power of $\eta$ with all component fields in $\mathcal{N}=4$ [52]. This can be done if we package all on-shell fields of $\mathcal{N}=4$ into a superfield or equivalently by applying the following rules

$$
\begin{aligned}
& g_{i}^{-} \sim \eta_{i}^{1} \eta_{i}^{2} \eta_{i}^{3} \eta_{i}^{4}, \quad \phi_{i}^{A B} \sim \eta_{i}^{A} \eta_{i}^{B}, \quad \Lambda_{i}^{A+} \sim \eta_{i}^{A}, \quad g_{i}^{+} \sim 1, \\
& \Lambda_{1}^{-} \sim-\eta_{i}^{2} \eta_{i}^{3} \eta_{i}^{4}, \quad \Lambda_{2 i}^{-} \sim \eta_{i}^{1} \eta_{i}^{3} \eta_{i}^{4}, \quad \Lambda_{3 i}^{-} \sim-\eta_{i}^{1} \eta_{i}^{2} \eta_{i}^{4}, \quad \Lambda_{4 i}^{-} \sim \eta_{i}^{1} \eta_{i}^{2} \eta_{i}^{3} .
\end{aligned}
$$

The analytic amplitudes are of degree- 8 and they are the elementary building blocks of the scalar graph approach. The next-to-minimal case are the amplitudes of degree-12 in $\eta$ which are obtained by connecting two analytic vertices [64] with a scalar propagator $1 / P^{2}$. Each analytic vertex contributes $8 \eta$ 's while a propagator removes $4 \eta$ 's. In fact, any $n$-point amplitude is characterised by a degree $8,12,16, \ldots,(4 n-8)$ and can be obtained from scalar diagrams with $1,2,3, \ldots$ analytic supervertices.

We now consider the fist non-minimal case of a diagram with two analytic supervertices (3.1) connected by a single scalar propagator. The diagram is depicted in figure 6 . The right supervertex has $n_{1}$ lines while the left one has $n_{2}$ lines in total, such that resulting amplitude $A_{n}$ has $n=n_{1}+n_{2}-2$ external lines. Suppressing summations over the distribution of $n_{1}$ and $n_{2}$ between the two supervertices, one can write down the expression for the amplitude of figure 6 as:

$$
\begin{aligned}
A_{n}= & \frac{1}{\prod_{l=1}^{n}\langle l l+1\rangle} \frac{1}{P_{I}^{2}} \frac{\left\langle j_{1} j_{1}+1\right\rangle\left\langle i_{1} i_{1}+1\right\rangle}{\left\langle i_{1} \bar{I}\right\rangle\left\langle\bar{I} j_{1}+1\right\rangle\left\langle j_{1} I\right\rangle\left\langle I i_{1}+1\right\rangle} \\
& \times \int \prod_{A=1}^{4} d \eta_{I}^{A} \delta^{(8)}\left(\lambda_{\bar{I} a} \eta_{I}^{A}+\sum_{l_{2} \neq \bar{I}}^{n_{2}} \lambda_{l_{2} a} \eta_{l_{2}}^{A}\right) \delta^{(8)}\left(\lambda_{I a} \eta_{I}^{A}+\sum_{l_{1} \neq I}^{n_{1}} \lambda_{l_{1} a} \eta_{l_{1}}^{A}\right) .
\end{aligned}
$$

The summations in the delta-functions arguments run over the $n_{1}-1$ external lines for right vertex, and $n_{2}-1$ external lines for the left one. The integration over the $4 \eta$ 's of the internal line arises because the two separate (unconnected) vertices in figure 6 would have $n_{1}+n_{2}$ lines and, hence, $n_{1}+n_{2}$ different $\eta$ 's while in the final amplitude there must be have $n=n_{1}+n_{2}-2 \eta$ 's. This is achieved by letting

$$
\eta_{\bar{I}}^{A}=\eta_{I}^{A},
$$


and integrating over $d^{4} \eta_{I}$. since the $I$ and $\bar{I}$ internal lines are connected by the propagator. The off-shell continuation of the internal spinors is defined as usual through,

$$
\lambda_{I a}=\sum_{l_{1} \neq I}^{n_{1}} p_{l_{1} a \dot{a}} \zeta^{\dot{a}}=-\lambda_{\bar{I} a} .
$$

The final step is to integrate out four $\eta_{I}$ 's by rearranging the arguments of the delta functions by use of $\int \delta\left(f_{2}\right) \delta\left(f_{1}\right)=\int \delta\left(f_{1}+f_{2}\right) \delta\left(f_{1}\right)$. Notice that the sum of two arguments, $f_{1}+f_{2}$, does not depend on $\eta_{I}$.

The final result of which we will make use in the following section is

$$
A_{n}=\frac{1}{\prod_{l=1}^{n}\langle l l+1\rangle} \delta^{(8)}\left(\sum_{i=1}^{n} \lambda_{i a} \eta_{i}^{A}\right) \prod_{A=1}^{4}\left(\sum_{l_{1} \neq I}^{n_{1}}\left\langle I l_{1}\right\rangle \eta_{l_{1}}^{A}\right) \frac{1}{D},
$$

and $D$ is the same as (2.4) used in sections 3 and 4 ,

$$
\frac{1}{D}=\frac{1}{P_{I}^{2}} \frac{\left\langle j_{1} j_{1}+1\right\rangle\left\langle i_{1} i_{1}+1\right\rangle}{\left\langle i_{1} I\right\rangle\left\langle I j_{1}+1\right\rangle\left\langle j_{1} I\right\rangle\left\langle I i_{1}+1\right\rangle} .
$$

As mentioned above, there are $12 \eta$ 's in the superamplitude (3.7). All component amplitudes of degree-12 can be obtained by expanding this expression in powers of $\eta$.

\subsection{Multi-soft limit of 2 scalars or 2 fermions forming a singlet and $m-2$ positive helicity gluons}

Based on the discussion of the last section it is now straightforward to obtain compact expressions for the leading behaviour of the multi-soft limits in two interesting cases. The first case concerns the multi-soft limit of two fermions forming a singlet and $m-2$ positive helicity gluons, while the second one is that of two scalars forming a singlet and $m-2$ positive helicity gluons in $N=4 \mathrm{SYM}$. The case of two scalars or the two fermions that do not form a singlet under SU(4) and no gluons had been treated recently in [25].

The fist step consists in identifying the relevant for the soft-limit diagrams. These are shown in figure 6 . The two scalars or fermions sit at the positions $i$ and $j$ while all other soft particles from 1 to $m$ are taken to be positive helicity gluons. As in the purely gluonic case the diagrams where two or more hard particles are attached to the same analytic supervertex with the negative helicity soft particle do not contribute in the leading order. One can now make use of (3.7) for each case separately. What is important is that only the diagrams of (6a) where both the fermions or the scalars are on the right supervertex can contribute. The diagrams (6b) and (6c) with one fermion or scalar per vertex are not present since the supervertex on the right can never be of degree 8 in the $\eta$ expansion as an analytic supervertex should be. This is so because all other external particles are taken to be positive helicity gluons which do not give any $\eta$ dependence. Notice that this is not the case for the diagram (6a) since there the right vertex is precisely of degree-8. Four $\eta$ s come from the two external fermions or scalars and another 4 from the negative helicity gluon propagator.

Consequently, what one has to do is to expand the product in (3.7) and keep the term proportional to $\eta_{i}^{A=1} \eta_{i}^{A=2} \eta_{j}^{A=3} \eta_{j}^{A=4}$ for the case of the two scalars sitting at positions $i$ 


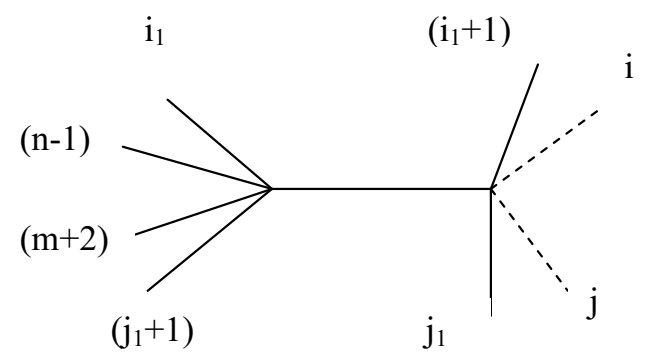

(6a)

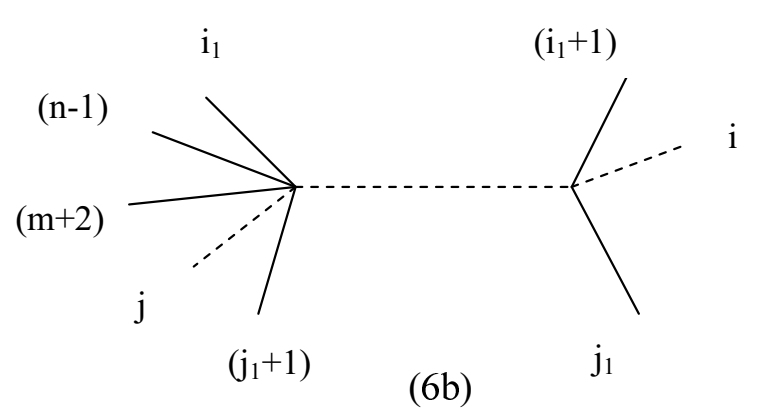

(6b)

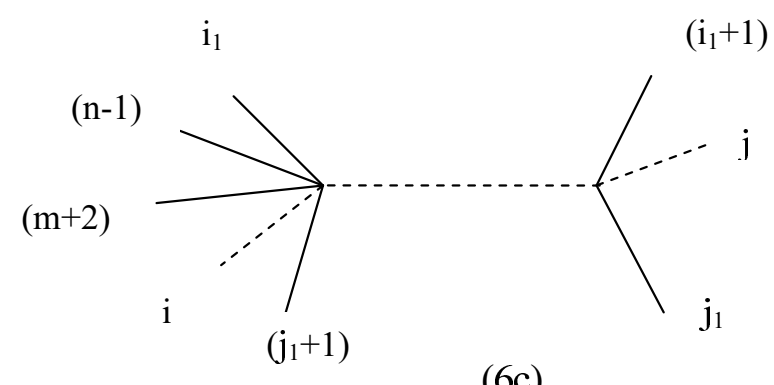

$(6 c)$

Figure 6. Diagrams relevant for the multi-soft limit of $\mathrm{m}$ particles 2 of which are scalars or fermions forming a singlet under $\mathrm{SU}(4)$ and sitting at positions $i$ and $j$. Notice that $i$ and $j$ is not needed to be adjacent. The class of diagrams on the left are the only which contribute. Diagrams (6b) and (6c) can not contribute because the right supervertex is not of degree- 8 . This is a consequence of the fact that all particles of the right vertex except the dashed ones are taken to be positive helicity gluons and as such do not give any $\eta$ dependence.

and $j$, i.e. $\phi_{i}^{12}$ and $\phi_{j}^{34}$. For the case of two fermions one should keep the term proportional either to $\eta_{i}^{A=1} \eta_{j}^{A=2} \eta_{j}^{A=3} \eta_{j}^{A=4}$ when the order of the fermions is $\Lambda_{i}^{A=1+} \Lambda_{A=1 j}^{-}$or to $\eta_{i}^{A=2} \eta_{i}^{A=3} \eta_{i}^{A=4} \eta_{j}^{A=1}$ when the order of the fermions is $\Lambda_{A=1 i}^{-} \Lambda_{j}^{A=1+}$. The computations resembles the gluonic case so we will omit the details. In all cases the final result takes the form

$$
A_{n} \rightarrow S_{m}(i, j) A_{n-m}
$$

where the multi-soft factor can be written in a unified form as

$$
S_{m}(i, j)=\frac{(-1)^{1-\theta(r-s)}\langle n m+1\rangle}{\prod_{q=n}^{m}\langle q q+1\rangle} \sum_{i_{1}=n-1}^{i-1} \sum_{j_{1}=j}^{\prime} \frac{\left\langle i^{-}\left|\not \phi_{i_{1}+1, j_{1}}\right| \zeta^{-}\right\rangle^{r}\left\langle j^{-}\left|\not \phi_{i_{1}+1, j_{1}}\right| \zeta^{-}\right\rangle^{s}}{D},
$$

with $D$ being precisely the quantity of (2.4) and $\theta(x)$ being the step function, $\theta(x)=1$ for $x \geq 0$ while $\theta(x)=0$ for $x<0$. When $r=s=2$ we have the case of the two scalars while the case $r=3, s=1$ corresponds to $\Lambda_{A=1 i}^{-} \Lambda_{j}^{A=1+}$ while the case $r=1, s=3$ corresponds to $\Lambda_{i}^{A=1+} \Lambda_{A=1 j}^{-}$. As in section 2 the prime over the sums is there to remind us that performing the sum we should omit the term with $i_{1}=n-1$ and $j_{1}=m+1$. As above the result (3.10) is independent of $\zeta$ which can be chosen to be $\zeta^{\dot{\alpha}}=\tilde{\lambda}_{1}^{\dot{\alpha}}$.

One important comment is in order. By counting the number of inverse power of $\delta$ it is easy to see that in all cases the soft factor diverges like $1 / \delta^{m}$, which is of the same order 


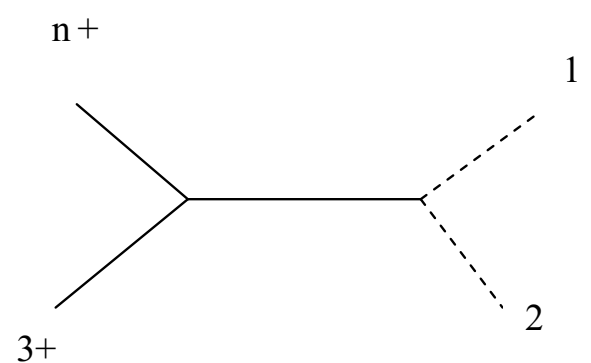

(7a)
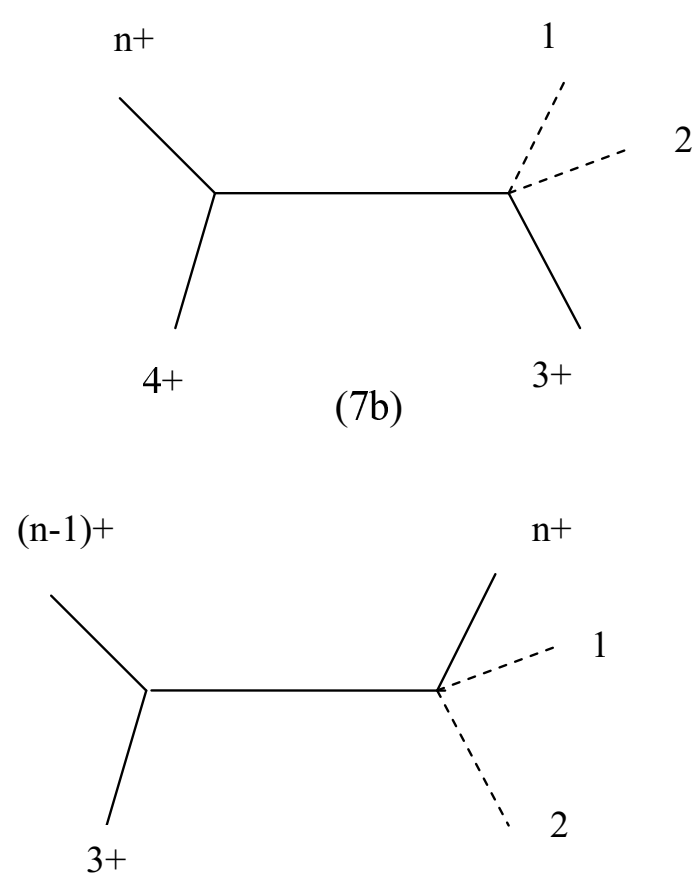

$(7 \mathrm{c})$

Figure 7. Diagrams contributing in the double-soft limit of 2 scalars or 2 fermions in a singlet configuration. Notice that as in figure 6 both particles should be on the same vertex.

as in the case of the multi-soft limit of one negative helicity gluon and $m-1$ gluons of positive helicity. This is quite natural from the point of view of the construction based on the analytic supervertex since all these cases fall in the same category where four $\eta \mathrm{s}$ are extracted from the external legs of the right supervertex.

\subsection{Double-soft limit of 2 scalars forming a singlet}

In this section, we consider the special case of the double-soft limit of two scalars which transform as a singlet under the $\mathrm{SU}(4) \mathrm{R}$-symmetry of $N=4 \mathrm{SYM}$. This particular case has been considered in [24]. Here, we will derive a simpler result that is in agreement with that of [24] (eq. (109)). As discussed at the end of the last section, this double-soft factor behaves as $1 / \delta^{2}$.

The result can be read from (3.10). For the sake of completeness we draw the set of contributing diagrams in figure 7 . There are three contributions from diagrams coming from (7a), (7b) and (7c). Let us start by writing down the contribution of (7c). By choosing the reference spinor to be $\zeta^{\dot{\alpha}}=\tilde{\lambda}_{1}^{\dot{\alpha}}$ we get from (3.10)

$$
S_{2}^{(7 c)}\left(\phi_{1}^{12}, \phi_{2}^{34}\right)=\frac{\langle n 3\rangle}{\langle n 1\rangle\langle 12\rangle\langle 23\rangle} \frac{\left\langle 1^{-}\left|\not \phi_{n}\right| 1^{-}\right\rangle^{2}\left\langle 2^{-}\left|\not p_{n}\right| 1^{-}\right\rangle^{2}\langle n-1 n\rangle\langle 23\rangle}{2 p_{n} \cdot q_{12}\left\langle 2^{-}\left|\not p_{n}\right| 1^{-}\right\rangle\left\langle 3^{-}\left|\not p_{n}\right| 1^{-}\right\rangle\left\langle n-1^{-}\left|\not p_{n}\right| 1^{-}\right\rangle\left\langle n^{-}\left|\not p_{12}\right| 1^{-}\right\rangle}
$$


After a bit of algebra this expression simplifies to

$$
S_{2}^{(7 c)}\left(\phi_{1}^{12}, \phi_{2}^{34}\right)=\frac{\langle n 1\rangle[n 1]}{2 p_{n} \cdot q_{12}\langle 12\rangle[21]} .
$$

Similarly, the diagram (7b) gives

$$
S_{2}^{(7 b)}\left(\phi_{1}^{12}, \phi_{2}^{34}\right)=\frac{\langle 13\rangle[31]}{2 p_{3} \cdot q_{12}\langle 12\rangle[21]} .
$$

Finally, our choice of the reference spinor $\zeta^{\dot{\alpha}}=\tilde{\lambda}_{1}^{\dot{\alpha}}$ gives zero for the diagram of (7a) since $S_{n}^{(7 a)}\left(\phi_{1}^{12}, \phi_{2}^{34}\right) \sim\left\langle 2^{-}\left|\phi_{12}\right| 1^{-}\right\rangle=0$. Overall we get

$$
S_{2}\left(\phi_{1}^{12}, \phi_{2}^{34}\right)=\frac{1}{\langle 12\rangle[21]}\left(\frac{\langle n 1\rangle[n 1]}{2 p_{n} \cdot q_{12}}+\frac{\langle 13\rangle[31]}{2 p_{3} \cdot q_{12}}\right) .
$$

One can check that (3.14) is a simpler representation of the double-soft factor of two scalars in a singlet configuration first obtained in [24]. As advertised above, this result scales as $1 / \delta^{2}$ in the double-soft limit.

\subsection{Double-soft limit of 2 fermions forming a singlet}

In a similar fashion it is straightforward to obtain the following result for the case of two soft fermions. The relevant diagram are again those of figure 7 with the only difference that now the dashed line denote fermions. Our choice of the reference spinor will again make the diagram of (7a) zero while the sum of the other two diagrams sum up to

$$
S_{2}\left(\Lambda_{1}^{A=1+} \Lambda_{A=12}^{-}\right)=-\frac{1}{\langle 12\rangle[21]}\left(\frac{\langle n 2\rangle[n 1]}{2 p_{n} \cdot q_{12}}+\frac{\langle 23\rangle[31]}{2 p_{3} \cdot q_{12}}\right) .
$$

As in the case of two scalars this result scales as $1 / \delta^{2}$ in the double-soft limit. This should be contrasted to the case of two fermions transforming non-trivially under the R-symmetry $\mathrm{SU}(4)$. In the latter case the amplitude behaves like $1 / \delta$ in the double-soft limit [25].

\subsection{Multi-soft limit of ( 2 fermions +1 scalar) and ( 4 fermions) forming a singlet}

We close this section by considering two last cases which can be easily extracted from the superamplitude (3.7). The first one is that of the triple-soft limit of a scalar $\phi_{1}^{12}$ sitting at position 1 and two positive helicity fermions at positions 2 and 3 , that is $\Lambda_{2}^{3+}$ and $\Lambda_{3}^{4+}$. The diagrams which contribute are similar to those of figure 7 with the only difference that there will be now three dashed lines instead of two denoting the 3 soft particles. Sticking with the same choice of the reference spinor as above and after a bit of spinor algebra we get

$$
\begin{gathered}
S_{3}\left(\phi_{1}^{12}, \Lambda_{2}^{A=3+}, \Lambda_{3}^{A=4+}\right)=\frac{1}{\langle 12\rangle\langle 23\rangle}\left(\frac{\langle 1 n\rangle\langle 2 n\rangle[n 1]}{2 p_{n} \cdot q_{13}\left\langle n^{-}\left|q_{2,3}\right| 1^{-}\right\rangle}-\frac{\langle 14\rangle\langle 24\rangle[41]}{2 p_{4} \cdot q_{13}\left\langle 4^{-}\left|q_{2,3}\right| 1^{-}\right\rangle}+\right. \\
\left.+\frac{\left\langle 1^{-}\left|q_{2,3}\right| 1^{-}\right\rangle\langle 23\rangle[31]\langle n 4\rangle}{q_{13}^{2}\left\langle 4^{-}\left|\not p_{2,3}\right| 1^{-}\right\rangle\left\langle n^{-}\left|\not p_{2,3}\right| 1^{-}\right\rangle}\right) .
\end{gathered}
$$


Similarly, for the case of the simultaneous soft limit of 4 positive helicity fermions we obtain

$$
\begin{aligned}
S_{4}\left(\Lambda_{1}^{1+}, \Lambda_{2}^{2+}, \Lambda_{3}^{3+}, \Lambda_{4}^{4+}\right)=\frac{1}{\langle 12\rangle\langle 23\rangle\langle 34\rangle} & \left(\frac{\langle 2 n\rangle\langle 3 n\rangle[n 1]}{2 p_{n} \cdot q_{14}\left\langle n^{-}\left|q_{2,4}\right| 1^{-}\right\rangle}-\frac{\langle 25\rangle\langle 35\rangle[51]}{2 p_{5} \cdot q_{14}\left\langle 5^{-}\left|q_{2,4}\right| 1^{-}\right\rangle}+\right. \\
& \left.+\frac{\left\langle 2^{-}\left|q_{3,4}\right| 1^{-}\right\rangle\left\langle 3^{-}\left|q_{2}+q_{4}\right| 1^{-}\right\rangle\langle n 5\rangle}{q_{14}^{2}\left\langle 5^{-}\left|q_{2,4}\right| 1^{-}\right\rangle\left\langle n^{-}\left|q_{2,4}\right| 1^{-}\right\rangle}\right) .
\end{aligned}
$$

Notice that the 4 -fermion and 1 scalar with 2 fermions soft factors scale as $1 / \delta^{4}$ and $1 / \delta^{3}$ respectively, in the multi-soft limit of (2.1). Finally, it is straightforward to write down the result for the multi-soft limit of 4 positive helicity fermions and any number of positive helicity gluons, as well as for the case of 1 scalar with 2 fermions and any number of positive helicity gluons along the lines of section 3.2.

\section{Conclusions}

In this present work we employ the MHV technique to show that scattering amplitudes with any number of consecutive soft particles behave universally in the multi-soft limit when all particles go soft simultaneously. In particular, we have shown how one can use the MHV diagrams to calculate the leading singularity of the simultaneous multi-soft limit of any number of consecutive particles. After identifying the diagrams which give the leading contribution we give the general rules from which one can immediately write down compact expressions for $m$ gluons, $k$ of which are negative helicity ones. As an example we explicitly give the expressions for the cases of $k$ being equal to 1 and 2 . In all cases the result takes the form of the multi-soft factor of $m$ positive helicity gluons times a function depending on the negative helicity ones.

Subsequently, we proceed to consider the case of amplitudes in $\mathcal{N}=4$ super Yang Mills theory. In this case, the scalar graph method has as building blocks the $\mathcal{N}=4$ analytic supervertices. Using this technique we obtain the multi-soft factor in the limit where 2 scalars or 2 fermions forming a singlet and $m-2$ positive helicity gluons become soft simultaneously. The double-soft limit of 2 scalars or 2 fermions forming a singlet gives an amplitude whose leading divergence is $1 / \delta^{2}$ and not $1 / \delta$ as in the case of 2 scalars or 2 fermions not forming a singlet under SU(4). As a bonus of the construction based on the analytic supervertices we also obtain expressions for the triple-soft limit of 1 scalar and 2 positive helicity fermions, as well as for the quadrapole-soft limit of 4 positive helicity fermions, in a singlet configuration. In all the cases we have considered the amplitude has a leading divergence of $1 / \delta^{m}$ in the soft limit (2.1). Finally, we think that it would be interesting to extend the present method to extract simple expressions for the subleading terms of the multi-soft factors considered in this article. Furthermore, the multi-soft expressions obtained in this work could be a more efficient starting point for reconstructing amplitudes by the inverse soft limit.

\section{Acknowledgments}

We thank George Savvidy for illuminating discussions. The work of the author was partially supported by the General Secretariat for Research and Technology of Greece and from the European Regional Development Fund MIS-448332-ORASY (NSRF 2007-13 ACTION, KRIPIS). 
Open Access. This article is distributed under the terms of the Creative Commons Attribution License (CC-BY 4.0), which permits any use, distribution and reproduction in any medium, provided the original author(s) and source are credited.

\section{References}

[1] F.E. Low, Bremsstrahlung of very low-energy quanta in elementary particle collisions, Phys. Rev. 110 (1958) 974 [INSPIRE].

[2] S. Weinberg, Photons and gravitons in S-matrix theory: derivation of charge conservation and equality of gravitational and inertial mass, Phys. Rev. 135 (1964) B1049 [INSPIRE].

[3] F. Cachazo and A. Strominger, Evidence for a new soft graviton theorem, arXiv:1404.4091 [INSPIRE].

[4] E. Casali, Soft sub-leading divergences in Yang-Mills amplitudes, JHEP 08 (2014) 077 [arXiv: 1404.5551] [INSPIRE].

[5] S.L. Adler, Consistency conditions on the strong interactions implied by a partially conserved axial vector current, Phys. Rev. 137 (1965) B1022 [INSPIRE].

[6] N. Arkani-Hamed, F. Cachazo and J. Kaplan, What is the simplest quantum field theory?, JHEP 09 (2010) 016 [arXiv:0808.1446] [INSPIRE].

[7] T. He, P. Mitra and A. Strominger, 2D Kac-Moody symmetry of 4D Yang-Mills theory, arXiv: 1503.02663 [INSPIRE].

[8] D. Kapec, V. Lysov, S. Pasterski and A. Strominger, Semiclassical Virasoro symmetry of the quantum gravity S-matrix, JHEP 08 (2014) 058 [arXiv: 1406.3312] [INSPIRE].

[9] T. He, V. Lysov, P. Mitra and A. Strominger, BMS supertranslations and Weinberg's soft graviton theorem, JHEP 05 (2015) 151 [arXiv:1401.7026] [INSPIRE].

[10] H. Bondi, M.G.J. van der Burg and A.W.K. Metzner, Gravitational waves in general relativity. 7. Waves from axisymmetric isolated systems,

Proc. Roy. Soc. Lond. A 269 (1962) 21 [INSPIRE].

[11] R.K. Sachs, Gravitational waves in general relativity. 8. Waves in asymptotically flat space-times, Proc. Roy. Soc. Lond. A 270 (1962) 103 [INSPIRE].

[12] G. Barnich and C. Troessaert, Symmetries of asymptotically flat 4 dimensional spacetimes at null infinity revisited, Phys. Rev. Lett. 105 (2010) 111103 [arXiv:0909.2617] [INSPIRE].

[13] G. Barnich and C. Troessaert, Supertranslations call for superrotations, PoS (CNCFG2010) 010 [Ann. U. Craiova Phys. 21 (2011) S11] [arXiv:1102.4632] [INSPIRE].

[14] G. Barnich and C. Troessaert, BMS charge algebra, JHEP 12 (2011) 105 [arXiv:1106. 0213] [INSPIRE].

[15] N. Beisert et al., Review of AdS/CFT integrability: an overview, Lett. Math. Phys. 99 (2012) 3 [arXiv:1012.3982] [InSPIRE].

[16] G. Georgiou, Two and three-point correlators of operators dual to folded string solutions at strong coupling, JHEP 02 (2011) 046 [arXiv:1011.5181] [INSPIRE].

[17] G. Georgiou, SL(2) sector: weak/strong coupling agreement of three-point correlators, JHEP 09 (2011) 132 [arXiv:1107.1850] [INSPIRE]. 
[18] G. Georgiou, V. Gili, A. Grossardt and J. Plefka, Three-point functions in planar $N=4$ super Yang-Mills theory for scalar operators up to length five at the one-loop order, JHEP 04 (2012) 038 [arXiv: 1201.0992] [INSPIRE].

[19] C. Durnford, G. Georgiou and V.V. Khoze, Instanton test of non-supersymmetric deformations of the $A d S_{5} \times S^{5}$, JHEP 09 (2006) 005 [hep-th/0606111] [INSPIRE].

[20] C.-S. Chu, G. Georgiou and V.V. Khoze, Magnons, classical strings and $\beta$-deformations, JHEP 11 (2006) 093 [hep-th/0606220] [INSPIRE].

[21] L.J. Dixon, Calculating scattering amplitudes efficiently, hep-ph/9601359 [INSPIRE].

[22] H. Elvang and Y.-T. Huang, Scattering amplitudes, arXiv:1308.1697 [INSPIRE].

[23] J.M. Henn and J.C. Plefka, Scattering amplitudes in gauge theories, Lect. Notes Phys. 883 (2014) 1 [INSPIRE].

[24] T. Klose, T. McLoughlin, D. Nandan, J. Plefka and G. Travaglini, Double-soft limits of gluons and gravitons, JHEP 07 (2015) 135 [arXiv: 1504.05558] [INSPIRE].

[25] A. Volovich, C. Wen and M. Zlotnikov, Double soft theorems in gauge and string theories, JHEP 07 (2015) 095 [arXiv: 1504.05559] [INSPIRE].

[26] W.-M. Chen, Y.-T. Huang and C. Wen, New fermionic soft theorems for supergravity amplitudes, Phys. Rev. Lett. 115 (2015) 021603 [arXiv:1412.1809] [INSPIRE].

[27] Z. Bern, S. Davies and J. Nohle, On loop corrections to subleading soft behavior of gluons and gravitons, Phys. Rev. D 90 (2014) 085015 [arXiv:1405.1015] [INSPIRE].

[28] S. He, Y.-T. Huang and C. Wen, Loop corrections to soft theorems in gauge theories and gravity, JHEP 12 (2014) 115 [arXiv:1405.1410] [INSPIRE].

[29] F. Cachazo and E.Y. Yuan, Are soft theorems renormalized?, arXiv:1405.3413 [INSPIRE].

[30] M. Bianchi, S. He, Y.-T. Huang and C. Wen, More on soft theorems: trees, loops and strings, arXiv: 1406.5155 [INSPIRE].

[31] J. Broedel, M. de Leeuw, J. Plefka and M. Rosso, Local contributions to factorized soft graviton theorems at loop level, Phys. Lett. B 746 (2015) 293 [arXiv:1411.2230] [INSPIRE].

[32] B.U.W. Schwab and A. Volovich, Subleading soft theorem in arbitrary dimensions from scattering equations, Phys. Rev. Lett. 113 (2014) 101601 [arXiv:1404.7749] [INSPIRE].

[33] N. Afkhami-Jeddi, Soft graviton theorem in arbitrary dimensions, arXiv:1405.3533 [INSPIRE].

[34] M. Zlotnikov, Sub-sub-leading soft-graviton theorem in arbitrary dimension, JHEP 10 (2014) 148 [arXiv:1407.5936] [INSPIRE].

[35] C. Kalousios and F. Rojas, Next to subleading soft-graviton theorem in arbitrary dimensions, JHEP 01 (2015) 107 [arXiv: 1407.5982] [INSPIRE].

[36] A.J. Larkoski, Conformal invariance of the subleading soft theorem in gauge theory, Phys. Rev. D 90 (2014) 087701 [arXiv: 1405.2346] [INSPIRE].

[37] F. Cachazo and E.Y. Yuan, Are soft theorems renormalized?, arXiv:1405.3413 [INSPIRE].

[38] J. Broedel, M. de Leeuw, J. Plefka and M. Rosso, Constraining subleading soft gluon and graviton theorems, Phys. Rev. D 90 (2014) 065024 [arXiv:1406.6574] [InSPIRE]. 
[39] Z. Bern, S. Davies, P. Di Vecchia and J. Nohle, Low-energy behavior of gluons and gravitons from gauge invariance, Phys. Rev. D 90 (2014) 084035 [arXiv:1406.6987] [InSPIRE].

[40] C.D. White, Diagrammatic insights into next-to-soft corrections, Phys. Lett. B 737 (2014) 216 [arXiv:1406.7184] [InSPIRE].

[41] M. Campiglia and A. Laddha, Asymptotic symmetries and subleading soft graviton theorem, Phys. Rev. D 90 (2014) 124028 [arXiv:1408.2228] [InSPIRE].

[42] Y.-J. Du, B. Feng, C.-H. Fu and Y. Wang, Note on soft graviton theorem by KLT relation, JHEP 11 (2014) 090 [arXiv: 1408.4179] [INSPIRE].

[43] H. Lüo, P. Mastrolia and W.J. Torres Bobadilla, Subleading soft behavior of QCD amplitudes, Phys. Rev. D 91 (2015) 065018 [arXiv:1411.1669] [INSPIRE].

[44] A. Sabio Vera and M.A. Vazquez-Mozo, The double copy structure of soft gravitons, JHEP 03 (2015) 070 [arXiv: 1412.3699] [INSPIRE].

[45] A.J. Larkoski, D. Neill and I.W. Stewart, Soft theorems from effective field theory, JHEP 06 (2015) 077 [arXiv: 1412.3108] [INSPIRE].

[46] M. Campiglia and A. Laddha, New symmetries for the gravitational S-matrix, JHEP 04 (2015) 076 [arXiv:1502.02318] [INSPIRE].

[47] R. Britto, F. Cachazo and B. Feng, New recursion relations for tree amplitudes of gluons, Nucl. Phys. B 715 (2005) 499 [hep-th/0412308] [INSPIRE].

[48] R. Britto, F. Cachazo, B. Feng and E. Witten, Direct proof of tree-level recursion relation in Yang-Mills theory, Phys. Rev. Lett. 94 (2005) 181602 [hep-th/0501052] [INSPIRE].

[49] F. Cachazo, S. He and E.Y. Yuan, Scattering of massless particles in arbitrary dimensions, Phys. Rev. Lett. 113 (2014) 171601 [arXiv:1307.2199] [INSPIRE].

[50] F. Cachazo, P. Svrček and E. Witten, MHV vertices and tree amplitudes in gauge theory, JHEP 09 (2004) 006 [hep-th/0403047] [INSPIRE].

[51] E. Witten, Perturbative gauge theory as a string theory in twistor space, Commun. Math. Phys. 252 (2004) 189 [hep-th/0312171] [INSPIRE].

[52] G. Georgiou and V.V. Khoze, Tree amplitudes in gauge theory as scalar MHV diagrams, JHEP 05 (2004) 070 [hep-th/0404072] [INSPIRE].

[53] G. Georgiou, E.W.N. Glover and V.V. Khoze, Non-MHV tree amplitudes in gauge theory, JHEP 07 (2004) 048 [hep-th/0407027] [rNSPIRE].

[54] A. Brandhuber, B.J. Spence and G. Travaglini, One-loop gauge theory amplitudes in $N=4$ super Yang-Mills from MHV vertices, Nucl. Phys. B 706 (2005) 150 [hep-th/0407214] [INSPIRE].

[55] J. Bedford, A. Brandhuber, B.J. Spence and G. Travaglini, A twistor approach to one-loop amplitudes in $N=1$ supersymmetric Yang-Mills theory, Nucl. Phys. B 706 (2005) 100 [hep-th/0410280] [INSPIRE].

[56] J. Bedford, A. Brandhuber, B.J. Spence and G. Travaglini, Non-supersymmetric loop amplitudes and MHV vertices, Nucl. Phys. B 712 (2005) 59 [hep-th/0412108] [INSPIRE].

[57] A. Brandhuber, B. Spence and G. Travaglini, From trees to loops and back, JHEP 01 (2006) 142 [hep-th/0510253] [INSPIRE]. 
[58] C. Quigley and M. Rozali, One-loop MHV amplitudes in supersymmetric gauge theories, JHEP 01 (2005) 053 [hep-th/0410278] [INSPIRE].

[59] L.F. Alday and J.M. Maldacena, Gluon scattering amplitudes at strong coupling, JHEP 06 (2007) 064 [arXiv:0705.0303] [INSPIRE].

[60] J.M. Drummond, J. Henn, G.P. Korchemsky and E. Sokatchev, On planar gluon amplitudes/Wilson loops duality, Nucl. Phys. B 795 (2008) 52 [arXiv:0709.2368] [INSPIRE].

[61] A. Brandhuber, P. Heslop and G. Travaglini, $M H V$ amplitudes in $N=4$ super Yang-Mills and Wilson loops, Nucl. Phys. B 794 (2008) 231 [arXiv:0707.1153] [InSPIRE].

[62] G. Georgiou, Null Wilson loops with a self-crossing and the Wilson loop/amplitude conjecture, JHEP 09 (2009) 021 [arXiv:0904.4675] [INSPIRE].

[63] F. Cachazo, P. Svrček and E. Witten, Twistor space structure of one-loop amplitudes in gauge theory, JHEP 10 (2004) 074 [hep-th/0406177] [INSPIRE].

[64] V.P. Nair, A current algebra for some gauge theory amplitudes, Phys. Lett. B 214 (1988) 215 [INSPIRE]. 